\title{
Constraints on the electroweak chiral Lagrangian from the precision data
}

\author{
Sukanta Dutta ${ }^{a, b}$, Kaoru Hagiwara ${ }^{b, c}$, Qi-Shu Yan ${ }^{d, e}$, and Kentaroh Yoshida ${ }^{f}$ \\ a SGTB Khalsa College, University of Delhi, Delhi-110007 India \\ ${ }^{b}$ KEK Theory Division, Tsukuba, 305-0801 Japan \\ ${ }^{c}$ The Graduate University for Advanced Studies (SOKENDAI), Tsukuba, 305-0801 Japan \\ ${ }^{d}$ Department of Physics, National Tsing Hua University, Hsinchu, Taiwan \\ e National Center of Theoretical Sciences (Theory Division), \\ 101, Section 2 Kuang Fu Road, Hsinchu, Taiwan and \\ ${ }^{f}$ Kavli Institute for Theoretical Physics, University of California, Santa Barbara, CA. 93106-4030, U.S.
}

\begin{abstract}
In the framework of the effective field theory method, we use the experimental data and the perturbative unitarity bounds to determine the values and uncertainty of all the 11 chiral coefficients $\left(\alpha_{i}, i=0, \cdots, 10\right)$ of the standard electroweak chiral Lagrangian. Up to linear terms in $\alpha_{i}$, we provide the one-loop renormalization group equations of all the chiral coefficients, which are calculated in the Feynman-'t Hooft gauge using the modified minimal subtraction scheme. With the improved renormalization group equations to sum over the logarithmic corrections, we analyze the current experimental uncertainty of oblique correction parameters, $S(\Lambda)$ and $T(\Lambda)$. We find that, due to the large uncertainty in the triple gauge-boson coupling measurements, the parameter space of positive $S(\Lambda)$ for $\Lambda>1 \mathrm{TeV}$ is still allowed by the current experimental data. $T(\Lambda)$ tends to increase with $\Lambda$ even in the presence of the operators that contribute to the triple and quartic gauge-boson couplings.
\end{abstract}

PACS numbers: 11.10.Gh, 11.10.Hi, 11.15.Ex, 10.30.Rd, 12.15.Ji, 12.15.Lk

\section{INTRODUCTION}

Electroweak symmetry breaking (EWSB) mechanism is the most important issue which will be explored at the Large Hadronic Collider (LHC). TeV-scale supersymmetric theories suggests that the electroweak symmetry is spontaneously broken by fundamental Higgs fields. On the contrary, QCD-like theories do not have fundamental scalar fields and suggest that the electroweak symmetry is dynamically broken by fermion-pair condensates [1, 2].

In order to obtain hints on the $\mathrm{TeV}$ scale physics that leads to the EWSB, it is useful to examine the consequence of the electroweak precision data of the bosonic sector in an integrated fashion. In this paper, we study the scale dependence of the coefficients of the electroweak chiral Lagrangian, and examine carefully if the present precision data exclude models with $S(\Lambda \sim 1 \mathrm{TeV})<0$ conclusively. We pay particular attention to the magnitude and sign of the coefficients of the operators that contribute only to the triple and quartic gauge-boson couplings and hence weakly constrained by the present data, because they enter in the $\beta$ functions of the running $S$ and $T$ parameters. Part of our findings have been reported in Ref. [3].

We follow the standard analysis of the chiral Lagrangian method [4, [5, [6] and include 11 operators up to mass dimension four in the electroweak chiral Lagrangian (EWCL) 7, 8, 9, 10]. This study extends the work of Bagger et. al. [11], who considered the effects of those operators that contribute to the weak boson two point functions only, i.e. 3 out of the 11 operators. We consider all the 11 operators, among which 5 operators contribute to the triple gauge boson couplings (TGCs) and 9 contribute to the quartic gauge boson couplings (QGCs).
We consider constraints from the TGC measurements at LEP2 [12, 13, 14, 15] and at the TeVatron [16] as well as those from the perturbative unitarity to bound the QGCs.

In the framework of the effective field theory method [17], all the 11 couplings in the EWCL are renormalization scale $\mu$ dependent quantities. Hence the electroweak precision data constrain the chiral coefficients at the low scale, $\mu=m_{Z}$. In order to find the connection between $S\left(m_{Z}\right)$ and $S(\Lambda)$, we extend the previous one-loop RGE [18] of the EWCL used by Bagger et. al. 11]. Along with the previous studies, we assume the validity of the perturbation theory and the absence of any additional resonances between $m_{Z}$ and $\Lambda$. The new $\beta$ functions takes account of all the the 11 chiral coefficients. The improved RGE makes it possible to analyze the effects of those operators which contribute only to the three-point and four-point gauge boson couplings on the uncertainty of $S(\Lambda)$ and $T(\Lambda)$ at the scale $\Lambda$ of new physics.

By utilizing the RGE to sum over the logarithmic corrections of quantum fluctuations and by analyzing the current experimental uncertainty of TGC and QGC, we find that in the most conservative perturbative calculation, the central value of $S(1 \mathrm{TeV})$ and its corresponding $1 \sigma$ error reads as

$$
S(1 \mathrm{TeV})=-0.02 \pm 0.20 .
$$

We observe that the current electroweak precision data, especially the data from the TGC measurements, have not reached the precision to fix the signs of $S(\Lambda)$ above $\Lambda>1 \mathrm{TeV}$. If we remove the effects of the chiral coefficients that contribute only to TGC and QGC, the central value and its $1 \sigma$ error reads as

$$
S(1 \mathrm{TeV})=-0.14 \pm 0.09,
$$


reproducing the result of Ref. [11].

In our analysis, we will use the formalism of nonlinear realization of EWSB, i.e. the gauged nonlinear $\sigma$ model. We consider the set of all the dimension 4 operators which are even under the transformation of discrete symmetries, $\mathrm{C}$ and $\mathrm{P}$. We omit all dimension 6 or higher order operators. There are works, for instance Ref. [19], which attempt to constrain the electroweak symmetry breaking models by including some of dimension 6 operators. By including more operators one can only make the allowed area of the $S(\Lambda)-T(\Lambda)$ plane larger, and hence our conclusion that $S(\Lambda=1 \mathrm{TeV})>0$ is still allowed persists.

This paper is organized as follows. In Sec. II, the bosonic sector of the EWCL in our analysis is introduced. In Sec. III, we briefly review the constraints on the chiral coefficients from the electroweak precision data on the gauge boson two point functions (A), those on the TGC (B), and those from the perturbative unitarity of the weak boson scattering amplitudes (C). In Sec. IV, we list the improved renormalization group equations (RGEs). In Sec. V, we study the experimental uncertainty of $S(\Lambda)-T(\Lambda)$ for $\Lambda=0.3 \mathrm{TeV}, 1 \mathrm{TeV}$, and $3 \mathrm{TeV}$ by using the improved RGEs. We close the paper in Sec. VI with discussions and conclusions. Two appendices are added to introduce our method to calculate the $\beta$ functions of the chiral coefficients (A) and the treatment of the ghost terms (B).

\section{THE OPERATORS IN OUR ANALYSIS}

In our analysis, we consider the following 14 bosonic operators which preserve discrete symmetries, $C$ and $P$ [7, 8, 9, 10]:

$$
\begin{aligned}
\mathcal{L}_{E W}= & -\frac{1}{g^{2}} \bar{H}_{1}-\frac{1}{g^{\prime 2}} \bar{H}_{2}-v^{2} \overline{\mathcal{L}}_{W / Z}+\alpha_{0} v^{2} \overline{\mathcal{L}}_{0} \\
& +\sum_{i=1}^{10} \alpha_{i} \overline{\mathcal{L}}_{i} \\
\bar{H}_{1}= & \frac{1}{2} \operatorname{tr}\left(W_{\mu \nu} W^{\mu \nu}\right) \\
\bar{H}_{2}= & \frac{1}{4} B_{\mu \nu} B^{\mu \nu} \\
\overline{\mathcal{L}}_{W / Z}= & \frac{1}{4} \operatorname{tr}\left(V_{\mu} V^{\mu}\right)
\end{aligned}
$$

where $g$ and $g^{\prime}$ are the gauge couplings of $S U(2)_{L}$ and $U(1)_{Y}$ gauge groups, respectively. The NambuGoldstone bosons are parameterized in the nonlinear form as

$$
U=\exp \left\{i \frac{2 \pi^{a} T^{a}}{v}\right\}
$$

The gauge covariant derivative, local gauge fields and their gauge covariant field strength are given as

$$
\begin{aligned}
V_{\mu} & =\left(\partial_{\mu} U\right) U^{\dagger}+i W_{\mu}-i U B_{\mu}^{Y} U^{\dagger} \\
W_{\mu} & =W_{\mu}^{a} T^{a} \\
B_{\mu}^{Y} & =B_{\mu} T^{3} \\
W_{\mu \nu} & =\partial_{\mu} W_{\nu}-\partial_{\nu} W_{\mu}+i\left[W_{\mu}, W_{\nu}\right] \\
B_{\mu \nu} & =\partial_{\mu} B_{\nu}-\partial_{\nu} B_{\mu}
\end{aligned}
$$

where $T^{a}=\tau^{a} / 2$, and $\tau^{a}$ are the Pauli matrices.

The couplings $\alpha_{i}$ form the 11 dimensional parameter space of the EWCL, where the corresponding operators $\mathcal{L}_{i}$ are given as

$$
\begin{aligned}
\overline{\mathcal{L}}_{0} & =\frac{1}{4}\left[\operatorname{tr}\left(\mathcal{T} V_{\mu}\right)\right]\left[\operatorname{tr}\left(\mathcal{T} V^{\mu}\right)\right], \\
\overline{\mathcal{L}}_{1} & =\frac{1}{2} B_{\mu \nu} \operatorname{tr}\left(\mathcal{T} W^{\mu \nu}\right), \\
\overline{\mathcal{L}}_{2} & =i \frac{1}{2} B_{\mu \nu} \operatorname{tr}\left(\mathcal{T}\left[V^{\mu}, V^{\nu}\right]\right), \\
\overline{\mathcal{L}}_{3} & =i \operatorname{tr}\left(W_{\mu \nu}\left[V^{\mu}, V^{\nu}\right]\right), \\
\overline{\mathcal{L}}_{4} & =\left[\operatorname{tr}\left(V_{\mu} V_{\nu}\right)\right]^{2}, \\
\overline{\mathcal{L}}_{5} & =\left[\operatorname{tr}\left(V_{\mu} V^{\mu}\right)\right]^{2} \\
\overline{\mathcal{L}}_{6} & =\operatorname{tr}\left(V_{\mu} V_{\nu}\right) \operatorname{tr}\left(\mathcal{T} V^{\mu}\right) \operatorname{tr}\left(\mathcal{T} V^{\nu}\right), \\
\overline{\mathcal{L}}_{7} & =\operatorname{tr}\left(V_{\mu} V^{\mu}\right)\left[\operatorname{tr}\left(\mathcal{T} V^{\nu}\right)\right]^{2}, \\
\overline{\mathcal{L}}_{8} & =\frac{1}{4}\left[\operatorname{tr}\left(\mathcal{T} W_{\mu \nu}\right)\right]^{2}, \\
\overline{\mathcal{L}}_{9} & =i \frac{1}{2} \operatorname{tr}\left(\mathcal{T} W_{\mu \nu}\right) \operatorname{tr}\left(\mathcal{T}\left[V^{\mu}, V^{\nu}\right]\right), \\
\overline{\mathcal{L}}_{10} & =\frac{1}{2}\left[\operatorname{tr}\left(\mathcal{T} V_{\mu}\right) \operatorname{tr}\left(\mathcal{T} V_{\nu}\right)\right]^{2},
\end{aligned}
$$

with

$$
\mathcal{T} \equiv 2 U T^{3} U^{\dagger}
$$

Each operator in the Lagrangian $\mathcal{L}_{E W}$ is invariant under the following local $S U(2)_{L} \times U(1)_{Y}$ gauge transformation

$$
\begin{aligned}
U & \rightarrow g_{L} U g_{Y}^{\dagger}, \\
W_{\mu} & \rightarrow g_{L} W_{\mu} g_{L}^{\dagger}-i g_{L} \partial_{\mu} g_{L}^{\dagger}, \\
W_{\mu \nu} & \rightarrow g_{L} W_{\mu \nu} g_{L}^{\dagger} \\
B_{\mu}^{Y} & \rightarrow B_{\mu}^{Y}-i g_{Y} \partial_{\mu} g_{Y}^{\dagger} \\
B_{\mu \nu} & \rightarrow B_{\mu \nu} \\
V_{\mu} & \rightarrow g_{L} V_{\mu} g_{L}^{\dagger} \\
\mathcal{T} & \rightarrow g_{L} \mathcal{T} g_{L}^{\dagger}
\end{aligned}
$$

where the gauge transformation factors $g_{L}$ and $g_{Y}$ are defined as

$$
\begin{aligned}
& g_{L}(x) \equiv \exp \left\{-i g \alpha^{a}(x) T^{a}\right\}, \\
& g_{Y}(x) \equiv \exp \left\{-i g^{\prime} \beta(x) T^{3}\right\} .
\end{aligned}
$$

Here $\alpha^{a}(x)$ and $\beta(x)$ are the real parameters of the gauge transformation of $S U(2)_{L}$ and $U(1)_{Y}$, respectively. 


\begin{tabular}{|c|c|c|c|}
\hline & 2 pt. vtx. & 3 pt. vtx. (TGC) & 4 pt. vtx. (QGC) \\
\hline$v^{2} \mathcal{L}_{0}$ & $\sqrt{ }$ & & \\
\hline $\mathcal{L}_{1}$ & $\sqrt{ }$ & $\sqrt{ }$ & \\
\hline $\mathcal{L}_{2}$ & & $\sqrt{ }$ & $\sqrt{ }$ \\
\hline $\mathcal{L}_{3}$ & & $\sqrt{ }$ & $\sqrt{ }$ \\
\hline $\mathcal{L}_{4}$ & & & $\sqrt{ }$ \\
\hline $\mathcal{L}_{5}$ & . & & $\sqrt{ }$ \\
\hline $\mathcal{L}_{6}$ & & & $\sqrt{ }$ \\
\hline $\mathcal{L}_{7}$ & & & $\sqrt{ }$ \\
\hline $\mathcal{L}_{8}$ & $\sqrt{ }$ & $\sqrt{ }$ & $\sqrt{ }$ \\
\hline $\mathcal{L}_{9}$ & & $\sqrt{ }$ & $\sqrt{ }$ \\
\hline$\overline{\mathcal{L}_{10}}$ & & & $\sqrt{ }$ \\
\hline
\end{tabular}

TABLE I: Operators and their contributions to 2, 3, and 4 point gauge boson vertices.

While operators $\overline{\mathcal{L}}_{W / Z}$ and $\overline{\mathcal{L}}_{0}$ contribute to the vector boson mass term, the operators $\overline{\mathcal{L}}_{i}, i=1, \cdots, 10$ contribute to their kinetic terms, TGC and QGC, as tabulated in Table प.

Accordingly we can classify the chiral coefficients into three groups: (1) $\alpha_{0}, \alpha_{1}$, and $\alpha_{8}$ contribute to the weak boson two-point functions, and are constrained by the electroweak precision data [23]; (2) $\alpha_{2}, \alpha_{3}$, and $\alpha_{9}$ contribute to the three-point couplings but not to the twopoint functions, and are constrained by the TGC measurements [12, 13, 14, 15]; (3) $\alpha_{4}, \alpha_{5}, \alpha_{6}, \alpha_{7}$, and $\alpha_{10}$ contribute only to the weak boson four-point couplings (QGC).

The typical size of the allowed range of 11 the chiral coefficients are hence:

$$
\begin{aligned}
O\left(\alpha_{0}, \alpha_{1}, \alpha_{8}\right) & \sim 10^{-3}, \\
O\left(\alpha_{2}, \alpha_{3}, \alpha_{9}\right) & \sim 10^{-1}, \\
O\left(\alpha_{4}, \alpha_{5}, \alpha_{6}, \alpha_{7}, \alpha_{10}\right) & \sim 1 .
\end{aligned}
$$

We will see in the following section, however, that the QGC couplings in Eq. (29) are constrained severely by the perturbation unitarity conditions for $\Lambda>1 \mathrm{TeV}$.

\section{CONSTRAINTS ON THE CHIRAL COEFFICIENTS}

If we do not consider a particular class of the underlying theory that leads to the electroweak symmetry breaking, all the chiral coefficients are arbitrary parameters. In this pure phenomenological viewpoint, we study constraints on their magnitude from the electroweak precision data of the gauge boson two-point functions, the TGC measurements, and from the perturbation unitarity conditions from the weak boson scattering amplitudes.

In our analysis, the bounds on the chiral coefficients listed in this section are determined at the scale $\mu=m_{Z}$. Strictly speaking, the constraints on $\alpha_{2}, \alpha_{3}$, and $\alpha_{9}$ from the TGC measurements at LEP2 are obtained at the scale $\mu \approx 2 m_{Z}$, and those on the $\alpha_{4}, \alpha_{5}, \alpha_{6}, \alpha_{7}$, and $\alpha_{10}$ are derived from the perturbative unitarity by assuming its validity up to the scale $\Lambda$. However, since we consider the effects of these loosely constrained chiral coefficients to the running of the most precisely measured coefficients, $\alpha_{0}, \alpha_{1}$, and $\alpha_{8}$ only, the scale dependence of all the other couplings give negligibly small effects on our results. They can be considered as higher order corrections of our leading order analysis.

Here we consider the most general case by retaining all the operators including those which violate the custodial $S U(2)_{c}$ symmetry, since the underlying dynamics can break it explicitly [20]. If we impose the custodial symmetry, the following chiral coefficients vanishes:

$$
\alpha_{0}, \alpha_{6}, \alpha_{7}, \alpha_{8}, \alpha_{9}, \alpha_{10} \equiv 0
$$

We discuss the implication of the custodial symmetry in the latter sections of this paper.

\section{A. Constraints from the two-point vertices}

By using three accurately measured quantities in Table III $1 / \alpha_{\mathrm{EM}}, G_{F}$, and $m_{Z}$, we fix the vacuum expectation value $v$, and the gauge couplings $g$ and $g^{\prime}$. The parameter $v$ is identified as

$$
v\left(m_{Z}\right)=1 / \sqrt{\sqrt{2} G_{F}}=246.26 \mathrm{GeV},
$$

and the two gauge couplings are identified as

$$
\begin{aligned}
g\left(m_{Z}\right) & =0.66, \\
g^{\prime}\left(m_{Z}\right) & =0.36,
\end{aligned}
$$

by using the tree-level relations. We retain only two digits in Eq. (32), and do not consider their errors, because their small variations do not affect our studies on the $S-T$ parameters in the leading order.

To make a global fit with the oblique parameters $S, T$, and $U$ 21], we follow the strategy of Peskin and Wells [22], and consider only three most precisely measured quantities, the average value of charged leptonic partial decay width $\Gamma_{\ell}$ of $Z$, the effective Weinberg mixing angle $\sin ^{2} \theta_{W}^{\text {eff }}$, and $m_{W}$. The latest results from the LEP, SLC, and Tevatron [24] are shown in Table III.

In the standard model, the relations among $\Gamma_{\ell}$, $\sin ^{2} \theta_{W}^{\text {eff }}, m_{W}$ and $S, T, U$ can be expressed as [25]

$$
\begin{array}{cc}
m_{W}(\mathrm{GeV})= & 80.377 \\
\Gamma_{\ell}(\mathrm{GeV})= & -0.288 \Delta S+0.418 \Delta T+0.337 \Delta U \\
\sin ^{2} \theta_{\mathrm{W}}^{\mathrm{eff}}= & -0.000018 \Delta S+0.00075 \Delta T \\
& +0.00359 \Delta S-0.00241 \Delta T
\end{array}
$$

where the central values are obtained by using the Zfitter 6.41 [26] with $m_{t}^{r e f}=175 \mathrm{GeV}, m_{H}^{r e f}=100 \mathrm{GeV}$, $\alpha_{s}\left(m_{Z}\right)=0.1176$, and $\delta \alpha_{5 h}=0.0279$ as inputs. The top 


\begin{tabular}{lc}
\hline \hline inputs & value \\
\hline $1 / \alpha_{\mathrm{EM}}\left(m_{Z}\right)$ & 127.87 \\
$G_{F}$ & $1.166 \times 10^{-5} \mathrm{GeV}^{-2}$ \\
$m_{Z}$ & $91.18 \mathrm{GeV}$ \\
\hline \hline
\end{tabular}

TABLE II: The inputs to fix $g\left(m_{Z}\right), g^{\prime}\left(m_{Z}\right)$, and $v\left(m_{Z}\right)$.

and Higgs mass dependence of the SM predictions are incorporated in the shifts $\Delta S, \Delta T$, and $\Delta U$ [25].

In order to utilize the above formula designed for the theories with a Higgs boson to theories like the EWCL without a Higgs boson, we follow the prescription of Bagger, Falk and Swartz, [1];

$$
\begin{aligned}
& \Delta S=\Delta S_{\mathrm{SM}}-S_{\mathrm{Higgs}}^{\mathrm{ND}}+S, \\
& \Delta T=\Delta T_{\mathrm{SM}}-T_{\mathrm{Higgs}}^{\mathrm{ND}}+T, \\
& \Delta U=\Delta U_{\mathrm{SM}}-U_{\mathrm{Higgs}}^{\mathrm{ND}}+U .
\end{aligned}
$$

Here $S, T$, and $U$, are the chiral couplings in the EWCL, which do not have dependence on $m_{H} . S_{\mathrm{Higgs}}^{\mathrm{ND}}, T_{\mathrm{Higgs}}^{\mathrm{ND}}$, and $U_{\mathrm{Higgs}}^{\mathrm{ND}}$, are the Higgs boson contributions in the heavy Higgs limit and can be simply expressed as

$$
\begin{aligned}
& S_{\mathrm{Higgs}}^{\mathrm{ND}}=-\frac{1}{6 \pi}\left[\frac{5}{12}-\ln \left(\frac{m_{H}}{m_{Z}}\right)\right], \\
& T_{\mathrm{Higgs}}^{\mathrm{ND}}=\frac{1}{\cos ^{2} \theta_{W}} \frac{3}{8 \pi}\left[\frac{5}{12}-\ln \left(\frac{m_{H}}{m_{Z}}\right)\right], \\
& U_{\mathrm{Higgs}}^{\mathrm{ND}}=0 .
\end{aligned}
$$

By using the parameterization of $\Delta S_{\mathrm{SM}}, \Delta T_{\mathrm{SM}}$, and $\Delta U_{\mathrm{SM}}$ in Ref. [25], we observe that the $m_{H}$ dependence cancel accurately for $m_{H}>300 \mathrm{GeV}$. For definiteness, we set $m_{H}=500 \mathrm{GeV}$ and find

$$
\begin{aligned}
\Delta S_{\mathrm{SM}}-S_{\mathrm{Higgs}}^{\mathrm{ND}}= & 0.057-0.007 x_{t}, \\
\Delta T_{\mathrm{SM}}-T_{\mathrm{Higgs}}^{\mathrm{ND}}= & -0.004+0.125 x_{t}+0.003 x_{t}^{2}, \\
\Delta U_{\mathrm{SM}}-U_{\mathrm{Higgs}}^{\mathrm{ND}}= & -0.003+0.022 x_{t},
\end{aligned}
$$

where $x_{t}=\left(m_{t}-175\right) / 10$ parameterize the remaining $m_{t}$ dependence.

It is now straightforward to find constraints of $S\left(m_{Z}\right)$, $T\left(m_{Z}\right)$, and $U\left(m_{Z}\right)$ from the data of Table III, by using the parameterizations Eqs. (33) 38), we find

$$
\begin{aligned}
& S\left(m_{Z}\right)=(-0.01 \pm 0.10) \\
& T\left(m_{Z}\right)=(+0.09 \pm 0.14) \\
& U\left(m_{Z}\right)=(+0.06 \pm 0.13)
\end{aligned}=\left(\begin{array}{ccc}
1 & & \\
0.93 & 1 & \\
-0.55 & -0.69 & 1
\end{array}\right),
$$

where the $1 \sigma$ errors and their correlations are given. In Fig. 1, we show the $1 \sigma(39 \%$ C.L. $)$ allowed region by a solid contour in the $S-T$ plane.

We can make one-to-one correspondences between the chiral coefficients $\alpha_{1}, \alpha_{8}, \alpha_{0}$ and the oblique parameters $S, T, U$ as in Ref. [10]

$$
\begin{aligned}
& \alpha_{1}(\mu) \equiv-\frac{1}{16 \pi} \quad S(\mu), \\
& \alpha_{0}(\mu) \equiv-\frac{\alpha_{\mathrm{EM}}}{2} T(\mu), \\
& \alpha_{8}(\mu) \equiv-\frac{1}{16 \pi} \quad U(\mu) .
\end{aligned}
$$

\begin{tabular}{lc}
\hline \hline parameter & current value \\
\hline$m_{W}$ & $80.403 \pm 0.029 \mathrm{GeV}$ \\
$\Gamma_{\ell}$ & $83.984 \pm 0.086 \mathrm{MeV}$ \\
$\sin ^{2} \theta_{W}^{\text {eff }}$ & $0.23152 \pm 0.00014$ \\
$m_{t}$ & $171.4 \pm 2.2 \mathrm{GeV}$ \\
\hline \hline
\end{tabular}

TABLE III: Current values of the three best measured electroweak parameters [24] and $m_{t}$ [27].

Here the magnitudes of $\alpha_{i}\left(m_{Z}\right)$ are fixed by $S, T$, and $U$ at $\mu=m_{Z}$, where $\alpha_{\mathrm{EM}}=1 / 137.36$ is the fine structure constant to conform with the $T$ parameter definition of by Peskin and Takeuchi[21]. The dependence of the $\alpha_{i}(\mu)$ parameters on $\mu$ are determined by the RGE provided in section IV. Eq. (40) can be interpreted as the definition of the running $S, T$, and $U$ parameters at $\mu \neq m_{Z}$. In terms of the chiral coefficients $\alpha_{1}\left(m_{Z}\right), \alpha_{0}\left(m_{Z}\right)$, and $\alpha_{8}\left(m_{Z}\right)$, the constraint Eq. (39) gives

$$
\begin{aligned}
& \alpha_{1}\left(m_{Z}\right)=(+0.02 \pm 0.20) \times 10^{-2} \\
& \alpha_{0}\left(m_{Z}\right)=(+0.03 \pm 0.05) \times 10^{-2} \\
& \alpha_{8}\left(m_{Z}\right)=(-0.12 \pm 0.25) \times 10^{-2}
\end{aligned}
$$

with the same correlation matrix.

When we impose the custodial symmetry. The constraint makes the allowed area smaller, and the optimal values of $S\left(m_{Z}\right)$ and $T\left(m_{Z}\right)$ moves up slightly, because of the positive value of $U\left(m_{Z}\right)$ in the 3-parameter fit and the negative correlations in the third row of Eq. (39). We obtain

$$
\begin{aligned}
& S\left(m_{Z}\right)=(+0.02 \pm 0.09) \\
& T\left(m_{Z}\right)=(+0.13 \pm 0.10)
\end{aligned} \rho=\left(\begin{array}{cc}
1 & \\
0.91 & 1
\end{array}\right) .
$$

The corresponding $1 \sigma$ contour is shown in FIG. 1 by a dotted-line contour. In terms of the chiral coefficients, we impost $\alpha_{8}\left(m_{Z}\right)=0$ and find

$$
\begin{aligned}
& \alpha_{1}\left(m_{Z}\right)=(-0.04 \pm 0.17) \times 10^{-2} \\
& \alpha_{0}\left(m_{Z}\right)=(+0.05 \pm 0.04) \times 10^{-2} .
\end{aligned}
$$

with the same correlation, $\rho=0.91$. We will use the constraints Eq. (42) or equivalently Eq. (43) in the analysis when the custodial $S U(2)_{c}$ symmetry is assumed.

These results roughly agree with those given in Ref. [11], where the small differences can be attributed to the changes in the input electroweak data.

\section{B. Constraints from the TGC}

There are three chiral coefficients $\alpha_{2}, \alpha_{3}, \alpha_{9}$ in the EWCL which contribute to the triple gauge boson couplings (TGC) but not to the two-point functions. The relations between the experimentally measured anomalous TGC [28] and the three-point chiral coefficients are 


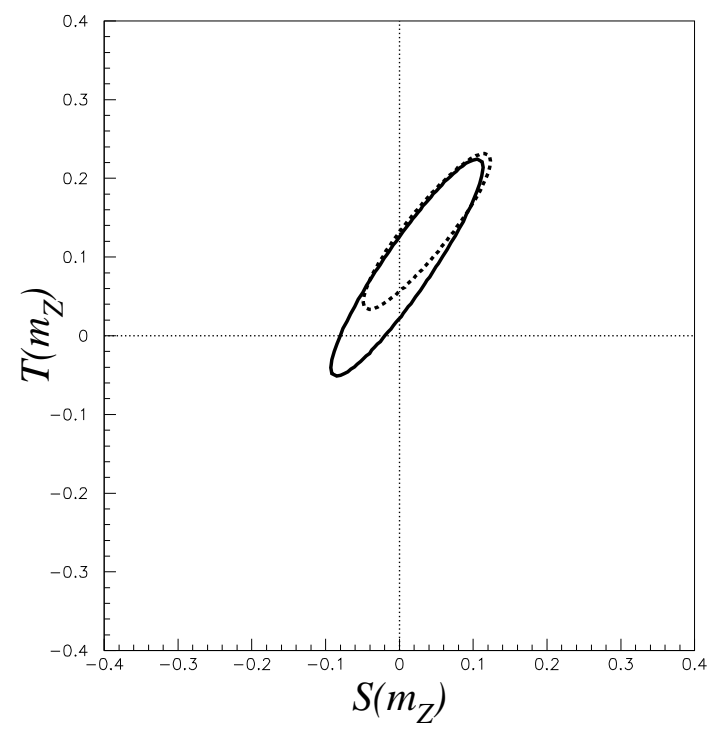

FIG. 1: The $S\left(m_{Z}\right)-T\left(m_{Z}\right)$ contours with $1 \sigma$ error from the electroweak data of Table III. The solid-line contour shows the constraint without the custodial symmetry while the dottedline contour shows the constraint with the custodial symmetry $\left(U\left(m_{Z}\right)=0\right)$.

given in Ref. [10]:

$$
\begin{aligned}
\delta k_{\gamma}= & \frac{e^{2}}{s^{2}}\left(-\alpha_{1}-\alpha_{8}+\alpha_{2}+\alpha_{3}+\alpha_{9}\right), \\
\delta k_{z}= & \frac{1}{c^{2}-s^{2}} \alpha_{0}+\frac{e^{2}}{c^{2}\left(c^{2}-s^{2}\right)} \alpha_{1}+\frac{e^{2}}{c^{2}}\left(\alpha_{1}-\alpha_{2}\right) \\
& +\frac{e^{2}}{s^{2}}\left(-\alpha_{8}+\alpha_{3}+\alpha_{9}\right), \\
\delta g_{Z}^{1}= & \frac{1}{c^{2}-s^{2}} \alpha_{0}+\frac{e^{2}}{c^{2}\left(c^{2}-s^{2}\right)} \alpha_{1}+\frac{e^{2}}{s^{2} c^{2}} \alpha_{3} .
\end{aligned}
$$

Because the constraints of $\alpha_{1}, \alpha_{0}$, and $\alpha_{8}$ in Eq. (41) are much more stringent than those of $\alpha_{2}, \alpha_{3}$, and $\alpha_{9}$ from the TGC measurements, we can simplify the above relations to

$$
\begin{aligned}
& \delta k_{\gamma}=\left(\alpha_{2}+\alpha_{3}+\alpha_{9}\right) g^{2} \\
& \delta k_{Z}=-\alpha_{2} g^{2}+\left(\alpha_{3}+\alpha_{9}\right) g^{2} \\
& \delta g_{1}^{Z}=\alpha_{3} g_{Z}^{2}
\end{aligned}
$$

in our analysis.

The unitarity bounds for anomalous TGC, $\delta k_{\gamma}, \delta k_{z}$ and $\delta g_{1}^{Z}$ derived [29] from processes $f^{1} f^{2} \rightarrow V^{1} V^{2}$ are summarized as

$$
\left|\delta k_{\gamma}\right|<\frac{1.86}{\Lambda^{2}},\left|\delta k_{Z}\right|<\frac{0.85}{\Lambda^{2}},\left|\delta g_{1}^{Z}\right|<\frac{0.87}{\Lambda^{2}} .
$$

Here $\Lambda(\mathrm{TeV})$ is the cut-off scale, up to which we request the validity of the perturbation theory. For $\Lambda=$ $1 \mathrm{TeV}$, these unitarity bounds constrain the magnitudes of anomalous TGC to be of order one. Hence, the unitarity constraints in Eq. (46) can be neglected when compared with the constraints from the TGC measurement even up to $\Lambda \sim 3 \mathrm{TeV}$.
Constraints from the D0 experiment at the Tevatron $p \bar{p}$ collider [16] on anomalous TGC are not much stronger than the unitarity bounds given in Eq. (46) at $1 \mathrm{TeV}$. It is the TGC measurements at LEP2 from the process $e^{+} e^{-} \rightarrow W^{+} W^{-}[12,13,14,15]$ that give the best constraints on these parameters. We use the constraints on $\alpha_{2}, \alpha_{3}$, and $\alpha_{9}$ from the LEP2 TGC measurements [12, 13, 14, 15] for the following three cases.

In case 1, we impose the custodial symmetry on the anomalous dimensionless EWCL couplings, rendering $\alpha_{9}=0$, i.e. Eq. (30). Eq. (45) leads to a relation among the three TGC observables,

$$
\delta \kappa_{Z}=-\delta \kappa_{\gamma} \tan ^{2} \theta_{w}+\delta g_{1}^{Z},
$$

In this scenario, we use the following results of the TGC measurements by the LEP working group 30], each of which has been obtained from one-parameter fit:

$$
\begin{aligned}
& \delta k_{\gamma}\left(m_{Z}\right)=-0.03 \pm 0.05, \\
& \delta g_{1}^{Z}\left(m_{Z}\right)=-0.02 \pm 0.02,
\end{aligned}
$$

Although there may be correlations between these two parameters in the two parameter fit, it can be small [30]. If we assume that the correlation between the errors is negligibly small, then from Eq. (48) and $\alpha_{9}=0$, we find

$$
\begin{aligned}
& \alpha_{2}\left(m_{Z}\right)=(-0.04 \pm 0.12) \\
& \alpha_{3}\left(m_{Z}\right)=(-0.03 \pm 0.04)
\end{aligned} \rho=\left(\begin{array}{cc}
1 & \\
-0.46 & 1
\end{array}\right) .
$$

In case 2, we adopt the two parameter-fitting result of the L3 collaboration [14], which has also been obtained under the custodial symmetry (47), and reads:

$$
\begin{aligned}
\delta k_{\gamma}\left(m_{Z}\right) & =(+0.16 \pm 0.13) \\
\delta g_{1}^{Z}\left(m_{Z}\right) & =(-0.09 \pm 0.05)
\end{aligned} \rho=\left(\begin{array}{cc}
1 & \\
-0.71 & 1
\end{array}\right) .
$$

In terms of the chiral coefficients, we find

$$
\begin{aligned}
& \alpha_{2}\left(m_{Z}\right)=(+0.54 \pm 0.36) \\
& \alpha_{3}\left(m_{Z}\right)=(-0.16 \pm 0.10)
\end{aligned} \rho=\left(\begin{array}{cc}
1 & \\
-0.82 & 1
\end{array}\right) .
$$

In the above two cases we set $\alpha_{9}=0$ by appealing to the custodial symmetry. In general, the custodial $S U(2)_{c}$ symmetry that may explain the smallness of $\alpha_{0}$ does not necessarily imply the suppression of $\alpha_{9}$. This motivates us to examine the third case where the chiral coefficients are analyzed in the absence of the constraint from Eq. (47). Since the experimental analysis for generic TGC without the constraint cannot be found, we use the measurement of $k_{Z}\left(m_{Z}\right)$ from the L3 collaboration [14] as an input, even though the experimental analysis was carried out by assuming the symmetry. Along with this analysis we choose the data on the other two TGC observables from the the LEP combined limits, Eq. (48), on $\delta k_{\gamma}\left(m_{Z}\right)$ and $\delta g_{1}^{Z}\left(m_{Z}\right)$ [30], as the case 3:

$$
\begin{aligned}
& \delta k_{Z}\left(m_{Z}\right)=-0.08 \pm 0.06 \\
& \delta k_{\gamma}\left(m_{Z}\right)=-0.03 \pm 0.05 \\
& \delta g_{1}^{Z}\left(m_{Z}\right)=-0.02 \pm 0.02
\end{aligned}
$$




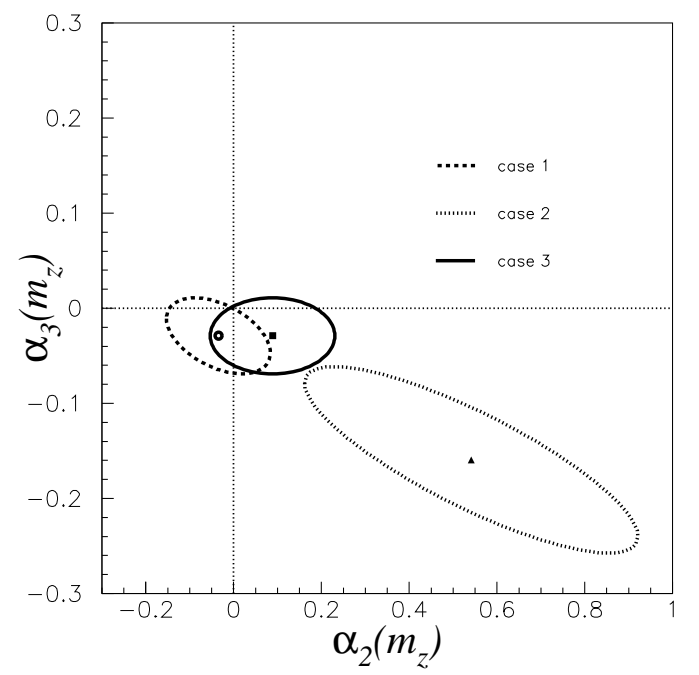

FIG. 2: The $\alpha_{2}-\alpha_{3}$ contours with $1 \sigma$ error (39\% confidence level). In case 1, the data is given in Eq. (48) and case $\mathbf{2}$, the data is given in Eq. (50). In both cases 1 and 2, the custodial symmetry condition $\alpha_{9}=0$ is imposed. In case 3, the data is given in Eq. (52) where $\alpha_{9}$ is taken as a free parameter in the fit.

Although the above limits should be much stronger than the true constraints when all the three TGC are allowed to vary freely, we adopt the above bounds as inputs of our general analysis without the custodial symmetry constraints. Again by neglecting the correlations among the errors of Eq. (52), we find

$$
\begin{aligned}
& \alpha_{2}\left(m_{Z}\right)=(+0.09 \pm 0.14) \\
& \alpha_{3}\left(m_{Z}\right)=(-0.03 \pm 0.04) \\
& \alpha_{9}\left(m_{Z}\right)=(-0.12 \pm 0.12)
\end{aligned} \rho=\left(\begin{array}{ccc}
1 & & \\
0.00 & 1 & \\
-0.65 & -0.32 & 1
\end{array}\right) \text {. }
$$

The bound is too stringent because the individual results of Eq. (52) are obtained under the constraint of Eq. (47) and also because the possible correlations among those measurements are neglected. However, it serves our purpose of showing the possible impacts of custodial $S U(2)_{c}$ symmetry violation.

In all the above three cases, we observe that $\alpha_{3}$ is more stringently constrained by the LEP2 data than $\alpha_{2}$ and $\alpha_{9}$. FIG. 2 shows the $1 \sigma$ error contour for $\alpha_{2}-\alpha_{3}$ for these three cases. We note that the central values of the L3 data deviate significantly from the prediction of the standard model.

\section{Perturbative unitarity constraints}

The most stringent theoretical constraint for the fourpoint chiral coefficients comes from the partial wave uni-

\begin{tabular}{|c|c|c|}
\hline \hline Chiral Coefficients & Central values & Error bars $( \pm)$ \\
\hline$\alpha_{0}\left(m_{Z}\right)$ & +0.0003 & 0.0005 \\
$\alpha_{1}\left(m_{Z}\right)$ & +0.0002 & 0.0020 \\
$\alpha_{2}\left(m_{Z}\right)$ & -0.09 & 0.14 \\
$\alpha_{3}\left(m_{Z}\right)$ & +0.03 & 0.04 \\
$\alpha_{4}\left(m_{Z}\right)$ & & $\sim \pi \frac{v^{4}}{\Lambda^{4}}$ \\
$\alpha_{5}\left(m_{Z}\right)$ & & $\sim \pi \frac{v^{4}}{\Lambda^{4}}$ \\
$\alpha_{6}\left(m_{Z}\right)$ & & $\sim \pi \frac{v^{4}}{\Lambda^{4}}$ \\
$\alpha_{7}\left(m_{Z}\right)$ & & $\sim \pi \frac{v^{4}}{\Lambda^{4}}$ \\
$\alpha_{8}\left(m_{Z}\right)$ & -0.0012 & 0.0025 \\
$\alpha_{9}\left(m_{Z}\right)$ & +0.13 & 0.12 \\
$\alpha_{10}\left(m_{Z}\right)$ & & $\sim \pi \frac{v^{4}}{\Lambda^{4}}$ \\
\hline \hline
\end{tabular}

TABLE IV: Summary on our knowledge of chiral coefficients, from Eq. (32), Eq. (41), Eq. (53) (here we take the most stringent bounds), and Eq. (54).

tarity conditions of the longitudinally polarized vector boson scattering amplitudes. These scattering amplitudes grow with energy if there are no new resonances up to the cut off scale $\Lambda$ [31].

Here we only consider the processes with $J=0$ channels and find

$$
\begin{aligned}
\left|4 \alpha_{4}+2 \alpha_{5}\right| & <3 \pi \frac{v^{4}}{\Lambda^{4}}, \\
\left|3 \alpha_{4}+4 \alpha_{5}\right| & <3 \pi \frac{v^{4}}{\Lambda^{4}} \\
\left|\alpha_{4}+\alpha_{6}+3\left(\alpha_{5}+\alpha_{7}\right)\right| & <3 \pi \frac{v^{4}}{\Lambda^{4}} \\
\left|2\left(\alpha_{4}+\alpha_{6}\right)+\alpha_{5}+\alpha_{7}\right| & <3 \pi \frac{v^{4}}{\Lambda^{4}}, \\
\left|\alpha_{4}+\alpha_{5}+2\left(\alpha_{6}+\alpha_{7}+\alpha_{10}\right)\right| & <\frac{6 \pi}{5} \frac{v^{4}}{\Lambda^{4}} .
\end{aligned}
$$

The five constraints are obtained from $W_{L}^{+} W_{L}^{+} \rightarrow$ $W_{L}^{+} W_{L}^{+}, W_{L}^{+} W_{L}^{-} \rightarrow W_{L}^{+} W_{L}^{-}, W_{L}^{+} W_{L}^{-} \rightarrow Z_{L} Z_{L}$, $W_{L}^{+} Z_{L} \rightarrow W_{L}^{+} Z_{L}$, and $Z_{L} Z_{L} \rightarrow Z_{L} Z_{L}$, respectively. In our analysis, the effects of the terms proportional to $v^{2} / \Lambda^{2}$ are found to be negligibly small and hence are dropped.

Recently, Distler et. al. [32] found that from the amplitudes of the scattering processes $Z_{L} Z_{L} \rightarrow Z_{L} Z_{L}$ and $W_{L} Z_{L} \rightarrow W_{L} Z_{L}$, by using dispersion relations and by assuming Lorentz invariance, analyticity, unitarity, and custodinal symmetry, it is possible to derive the lower bounds for the chiral parameters $\alpha_{4}$ and $\alpha_{5}$, which read

$$
\begin{aligned}
\alpha_{5}+2 \alpha_{4} & \geq \frac{1}{96 \pi^{2}} \times 1.08 \\
\alpha_{4} & \geq \frac{1}{96 \pi^{2}} \times 0.31
\end{aligned}
$$

To quote these number, we set $\mu=v$ and $\delta=1 / 5$.

We now summarize the current bounds on the chiral coefficients of the EWCL in Table IV. Among the six terms that contribute to the two-point functions, the first three coefficients, $g, g^{\prime}$, and $v$, are fixed by $\alpha_{\mathrm{EM}}\left(m_{Z}\right)$, $G_{F}$, and $m_{Z}$ in Table II, and the remaining three chiral coefficients, $\alpha_{0}, \alpha_{1}$, and $\alpha_{8}$ are constrained from the $\mathrm{Z}$ pole data, the precise $\mathrm{W}$ mass measurement, and the top quark mass measurement, see Table III. The three three-point chiral coefficients, $\alpha_{2}, \alpha_{3}$, and $\alpha_{9}$ are determined from the LEP2 W pair production measurements, and the five four-point chiral coefficients, $\alpha_{4}, \alpha_{5}, \alpha_{6}, \alpha_{7}$, 
and $\alpha_{10}$ are constrained by the five perturbative unitarity conditions of Eq. (54) if there is no new resonances up to the scale $\Lambda$.

We observe from Table IV that the two-point coefficients $\alpha_{0}, \alpha_{1}, \alpha_{8}$ are constrained to be $\sim 10^{-3}$, the TGC coefficients $\alpha_{2}, \alpha_{3}$, and $\alpha_{9}$ can be $\sim 10^{-1}$, while the remaining coefficients should be smaller than $\pi(v / \Lambda)^{4}$, or $10^{-2}$ for $\Lambda \sim 1 \mathrm{TeV}$.

It may be instructive to compare the above constraints with the corresponding ones of the QCD chiral theory. Reference [5] presents the fit of these dimensionless couplings in the chiral perturbation theory describing low energy QCD. The constraints found in reference [6] are $L_{9}\left(m_{\eta}\right)=\left(7.4 \pm 0.7 \times 10^{-3}\right)$ and $L_{10}\left(m_{\eta}\right)=$ $\left(-6.0 \pm 0.7 \times 10^{-3}\right)$, which correspond to $\alpha_{2}\left(=\alpha_{3}\right)$ and $\alpha_{1}$ respectively, in the electroweak theory.

The large and positive value of $\alpha_{1}$ at $\Lambda \sim 1 \mathrm{TeV}$, favored by the electroweak data, has been confronted against the large and negative value of $L_{10}\left(m_{\eta}\right)$, which led to the assertion [21] that the electroweak symmetry breaking does not mimic QCD. Although strictly QCD-like theories may also give $\alpha_{2} \sim \alpha_{3} \sim 10^{-2}$ as $L_{9}\left(m_{\eta}\right)$, we examine possible implications of models with $\alpha_{2}, \alpha_{3}, \alpha_{9} \sim 10^{-1}$, which are still allowed by the present TGC measurements.

\section{THE $\beta$ FUNCTION OF CHIRAL COEFFICIENTS AT ONE-LOOP LEVEL}

In order to find the connection between $S\left(m_{Z}\right)-$ $T\left(m_{Z}\right)$ and $S(\Lambda)-T(\Lambda)$, by using the method developed in [33], we extend the previous one-loop RGE [18] of the EWCL by including the three-point and four-point chiral coefficients in the $\beta$ functions of $S$ and $T$ (equivalently, the $\beta$ functions of the chiral coefficients $\alpha_{1}$ and $\left.\alpha_{0}\right)$. In the Wilsonian renormalization group concept, at the one-loop level, all dimensionless chiral coefficients $\alpha_{i}$ should run in a logarithmic way from $m_{Z}$ to $\Lambda$, just like the gauge couplings, due to the screening effects of the active quantum degree of freedoms (such as the Goldstone particles, the gauge bosons, and the ghosts). This logarithmic running is obtained by using the dimensional regularization in our calculation.

The RGE for the gauge couplings $g$ and $g^{\prime}$, for the vev term $v^{2}$, as well as for the chiral coefficients $\alpha_{i}$ can be simply expressed as

$$
\begin{aligned}
\frac{d}{d t} g & =\frac{1}{8 \pi^{2}} \beta_{g}, \\
\frac{d}{d t} g^{\prime} & =\frac{1}{8 \pi^{2}} \beta_{g^{\prime}}, \\
\frac{d}{d t} v^{2} & =\frac{1}{8 \pi^{2}} \beta_{v^{2}}, \\
\frac{d}{d t} \alpha_{i} & =\frac{1}{8 \pi^{2}} \beta_{\alpha_{i}},
\end{aligned}
$$

Where $t=\ln \left(\mu / m_{Z}\right)$. The $\beta_{g, g^{\prime}, v^{2}, \alpha_{i}}$ are the beta functions for the running of $g$ and $g^{\prime}, v^{2}$, as well as $\alpha_{i}$. Below we list the $\beta$ functions of the chiral coefficients of the EWCL at one-loop. Technical details of our calculation is described in Appendix A. The gauge fixing terms and the treatment of the ghost terms are presented in Appendix B.

We organize and group these $\beta$ functions in the order of their contributions to the multi point functions. The $\beta$ functions are written for those operators which contribute to the two point functions $\left(\alpha_{0}, \alpha_{1}\right.$, and $\left.\alpha_{8}\right)$, three point functions $\left(\alpha_{2}, \alpha_{3}\right.$, and $\left.\alpha_{9}\right)$, and four point functions $\left(\alpha_{4}, \alpha_{5}, \alpha_{6}, \alpha_{7}\right.$, and $\left.\alpha_{10}\right)$. In the standard derivative power counting rule, these terms are counted as $O\left(p^{6}\right)$ order effects.

We first write $\beta_{g, g^{\prime}}$ for the gauge couplings.

$$
\begin{gathered}
\beta_{g}=\frac{g^{3}}{2}\left[-\frac{29}{4}-\frac{\alpha_{0}}{6}-\alpha_{1} g^{2}-4 \alpha_{8} g^{2}\right. \\
\left.+\frac{5 \alpha_{2} g^{\prime 2}}{6}-\alpha_{3}\left(\frac{28 g^{2}}{3}+\frac{g^{\prime 2}}{2}\right)-\frac{13 \alpha_{9} g^{2}}{6}\right] \\
\beta_{g^{\prime}}=\frac{g^{\prime 3}}{2}\left(\frac{1}{12}-\frac{\alpha_{0}}{3}-2 \alpha_{1} g^{2}\right. \\
\left.-\alpha_{2} g^{2}+\frac{5 \alpha_{3} g^{2}}{3}\right)
\end{gathered}
$$

It is noted that the $\beta_{g}$ and $\beta_{g^{\prime}}$ both have constant terms which are expected by naively counting of the active bosonic degrees of freedom. Compared to the SM $\beta$ functions, only the physical Higgs boson contributions are absent. We have not included the contribution of active fermionic degree of freedoms which can be fixed in a straightforward manner. Also we find that the threepoint chiral coefficients $\alpha_{2}, \alpha_{3}$, and $\alpha_{9}$, contribute to the $\beta$ functions of the gauge couplings, and can modify the asymptotic behavior of gauge couplings, which should match the gauge couplings of the ultra-violet theory at the scale $\Lambda$.

The $\beta$ function of $v^{2}$ is given as

$$
\begin{aligned}
\beta_{v^{2}}= & \frac{3 g^{2}}{2}+\frac{3 g^{\prime 2}}{4}+\alpha_{0}\left(\frac{3}{2} g^{2}-3 g^{\prime 2}\right) \\
& -\frac{3 \alpha_{1} g^{2} g^{\prime 2}}{2}+\frac{3 \alpha_{8} g^{4}}{4} \\
& +\alpha_{2}\left(3 g^{2} g^{\prime 2}-\frac{3 g^{\prime 4}}{2}\right)-\alpha_{3}\left(2 g^{4}-3 g^{2} g^{\prime 2}\right) \\
& -\alpha_{9}\left(g^{4}-\frac{3 g^{2}{g^{\prime}}^{2}}{2}\right) \\
& -\alpha_{4}\left(\frac{21}{4} g^{4}+\frac{3 g_{Z}^{4}}{4}\right)-\alpha_{5}\left(\frac{15}{2} g^{4}+3 g_{Z}^{4}\right) \\
& -\frac{3 \alpha_{6} g_{Z}^{4}}{4}-3 \alpha_{7} g_{Z}^{4} .
\end{aligned}
$$

For $\beta_{v^{2}}$ we notice that it has no dependence on $\alpha_{10}$. This can be attributed to the fact that the use of dimensional regularization results in retaining only the logarithmic running of $v^{2}$. 
The $\beta$ functions of chiral couplings, $\alpha_{0}, \alpha_{1}$, and $\alpha_{8}$, are given as

$$
\begin{aligned}
\beta_{\alpha_{0}}= & \frac{3 g^{\prime 2}}{8}+\alpha_{0}\left(\frac{9 g^{2}}{4}-\frac{9 g^{\prime 2}}{4}\right) \\
& -\frac{3 \alpha_{1} g^{2} g^{\prime 2}}{4}+\frac{3 \alpha_{8} g^{4}}{8} \\
& +\alpha_{2}\left(\frac{3 g^{2} g^{\prime 2}}{2}-\frac{3 g^{\prime 4}}{4}\right)+\frac{3 \alpha_{3} g^{2} g^{\prime 2}}{2} \\
& +\alpha_{9}\left(-\frac{g^{4}}{2}+\frac{3 g^{2} g^{\prime 2}}{4}\right) \\
& +\alpha_{4}\left(\frac{15 g^{2} g^{\prime 2}}{4}+\frac{15 g^{\prime 4}}{8}\right)+\alpha_{5}\left(\frac{3 g^{2} g^{\prime 2}}{2}+\frac{3 g^{\prime 4}}{4}\right) \\
& +\alpha_{6}\left(\frac{3 g^{4}}{4}+\frac{33 g_{Z}^{4}}{8}\right)+\alpha_{7}\left(3 g^{4}+3 g_{Z}^{4}\right) \\
& +\frac{9 \alpha_{10} g_{Z}^{4}}{2} \cdot \\
& \beta_{\alpha_{1}}=\frac{1}{12}+4 \alpha_{1} g^{2}-\alpha_{8} g^{2} \\
& -\frac{5 \alpha_{2} g^{2}}{2}+\frac{5 \alpha_{3} g^{2}}{6}-\frac{\alpha_{9} g^{2}}{2}, \\
& \beta_{\alpha_{8}}=\frac{\alpha_{0}}{2}+\alpha_{1} g^{\prime 2}+12 \alpha_{8} g^{2} \\
& -\frac{5 \alpha_{2} g^{\prime 2}}{6}+\frac{\alpha_{3} g^{\prime 2}}{2}-\frac{17 \alpha_{9} g^{2}}{6}
\end{aligned}
$$

We remark here that the three-point chiral coefficients $\alpha_{2}, \alpha_{3}$, and $\alpha_{9}$, affect the running behavior of the twopoint chiral coefficients $\alpha_{1}, \alpha_{8}$, and $\alpha_{0}$, parameters. We observe that the $\beta$ functions for $\alpha_{1}$ and $\alpha_{8}$ do not contain the four-point chiral coefficients $\alpha_{4}, \alpha_{5}, \alpha_{6}, \alpha_{7}$, and $\alpha_{10}$ but $\alpha_{0}$ does. Interpreting this exception in term of Feynman diagrams, we observe that $\alpha_{1}$ and $\alpha_{8}$ only receives the radiative corrections through the diagram of FIG. (3a) while $\alpha_{0}$ receives the radiative corrections from both FIG. (3a) and FIG. (3b). It is the diagram FIG. $(3 \mathrm{~b})$ which renders the entry of the four-point chiral coefficients in the $\beta$ function of $\alpha_{0}$.

The $\beta$ functions of the three-point chiral coefficients $\alpha_{2}, \alpha_{3}$ and $\alpha_{9}$, are given as

$$
\begin{aligned}
\beta_{\alpha_{2}}= & \frac{1}{24}+\frac{\alpha_{0}}{6}-\frac{\alpha_{1} g^{2}}{2} \\
& +\alpha_{2}\left(2 g^{2}+\frac{g^{\prime 2}}{12}\right)+\frac{5 \alpha_{3} g^{2}}{12}-\frac{\alpha_{9} g^{2}}{12} \\
& +\alpha_{4}\left(\frac{g^{2}}{4}+\frac{g^{\prime 2}}{2}\right)-\alpha_{5}\left(\frac{g^{2}}{2}-g^{\prime 2}\right) \\
& -\frac{\alpha_{6} g^{\prime 2}}{2}-\alpha_{7} g^{\prime 2}
\end{aligned}
$$

$$
\begin{aligned}
\beta_{\alpha_{3}}= & \frac{1}{24}-\frac{\alpha_{0}}{6}-\frac{\alpha_{1} g^{\prime 2}}{4} \\
& +\frac{\alpha_{2} g^{\prime 2}}{6}+\alpha_{3}\left(\frac{59 g^{2}}{12}+\frac{g^{\prime 2}}{4}\right)-\frac{5 \alpha_{9} g^{2}}{3} \\
& +\alpha_{4}\left(\frac{5 g^{2}}{4}+\frac{3 g^{\prime 2}}{8}\right)-\alpha_{5}\left(\frac{5 g^{2}}{2}-\frac{g^{\prime 2}}{4}\right) \\
& +\alpha_{6}\left(\frac{5 g^{2}}{4}+\frac{3 g^{\prime 2}}{8}\right)-\alpha_{7}\left(\frac{5 g^{2}}{2}-\frac{g^{\prime 2}}{4}\right), \\
\beta_{\alpha_{9}}= & \frac{\alpha_{0}}{2}-\frac{\alpha_{1} g^{\prime 2}}{4}-\frac{3 \alpha_{8} g^{2}}{4} \\
+ & \frac{\alpha_{2} g^{\prime 2}}{12}+\frac{5 \alpha_{3} g^{\prime 2}}{4}+\frac{119 \alpha_{9} g^{2}}{12} \\
& -\frac{7 \alpha_{4} g^{\prime 2}}{8}-\frac{5 \alpha_{5} g^{\prime 2}}{4} \\
& -\alpha_{6}\left(\frac{5 g^{2}}{4}+\frac{7 g^{\prime 2}}{8}\right)-\alpha_{7}\left(-\frac{5 g^{2}}{2}+\frac{5 g^{\prime 2}}{4}\right)
\end{aligned}
$$

The $\beta$ functions of the four-point chiral coefficients, $\alpha_{4}$, $\alpha_{5}, \alpha_{6}, \alpha_{7}$, and $\alpha_{10}$ are given as

$$
\begin{aligned}
\beta_{\alpha_{4}}= & -\frac{1}{12}-\alpha_{0}-\frac{5 \alpha_{2} g^{\prime 2}}{6}-\frac{\alpha_{3} g^{2}}{2}-\frac{\alpha_{9} g^{2}}{6} \\
& +\alpha_{4}\left(\frac{11 g^{2}}{2}+6{g^{\prime}}^{2}\right)+\alpha_{5}\left(2 g^{2}+3 g^{\prime 2}\right) \\
& +\alpha_{6}\left(-\frac{g^{2}}{2}+\frac{7 g^{\prime 2}}{2}\right)+2 \alpha_{7} g^{\prime 2} \\
\beta_{\alpha_{5}}=- & \frac{1}{24}+\frac{\alpha_{0}}{2}-\frac{\alpha_{2} g^{\prime 2}}{3}-\frac{\alpha_{3} g^{2}}{2}-\frac{\alpha_{9} g^{2}}{3} \\
& -\alpha_{4}\left(g^{2}+\frac{15 g^{\prime 2}}{4}\right)+\alpha_{5}\left(g^{2}-\frac{3 g^{\prime 2}}{2}\right) \\
& -\alpha_{6}\left(\frac{g^{2}}{4}+\frac{3 g^{\prime 2}}{4}\right)+\alpha_{7}\left(-\frac{3 g^{2}}{2}+\frac{3 g^{\prime 2}}{2}\right) \\
\beta_{\alpha_{6}}= & \frac{3 \alpha_{0}}{2}-\alpha_{2} g^{\prime 2}+\frac{25 \alpha_{4} g^{\prime 2}}{4}+\frac{7 \alpha_{5} g^{\prime 2}}{2} \\
& -\alpha_{6}\left(\frac{25 g^{2}}{4}-\frac{5 g^{\prime 2}}{4}\right)+2 \alpha_{7} g^{2} \\
+ & +\alpha_{10}\left(-g^{2}+5 g^{\prime 2}\right) \\
\beta_{\alpha_{7}}=- & \frac{3 \alpha_{0}}{4}-\frac{3 \alpha_{2} g^{\prime 2}}{4}+\frac{\alpha_{9} g^{\prime 2}}{4} \\
+ & \frac{41 \alpha_{4} g^{\prime 2}}{8}++\frac{11 \alpha_{5} g^{\prime 2}}{4} \\
+ & \left(-\frac{5 g^{2}}{8}+\frac{33 g^{\prime 2}}{8}\right)+\alpha_{7}\left(\frac{13 g^{2}}{4}+\frac{7 g^{\prime 2}}{4}\right) \\
&
\end{aligned}
$$




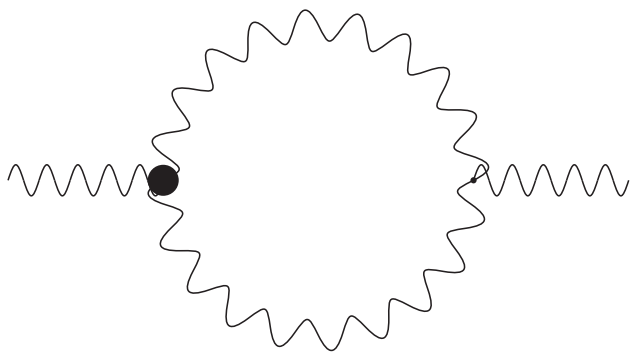

(3a)

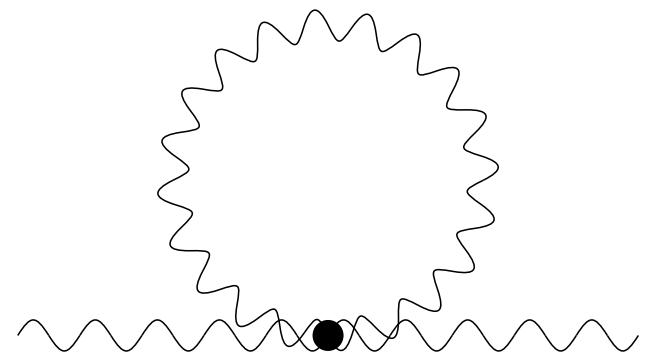

(3b)

FIG. 3: Feynman diagrams for two-point functions. Wavy line loops should include vector, Goldstone, and ghost loops. The solid circles show the chiral operators $\mathcal{L}_{i}$.

$$
\beta_{\alpha_{10}}=\frac{17 \alpha_{6}{g^{\prime 2}}^{2}}{4}+\frac{7 \alpha_{7} g^{\prime 2}}{2}+9 \alpha_{10}\left(g^{2}-g^{\prime 2}\right)
$$

In calculating the above $\beta$ functions, we have performed the following consistency checks: (1) We ensure that when all the anomalous couplings are set to vanish, our calculation reduces not only to the standard $\beta$ functions for gauge couplings but also to the constant terms in the $\beta$ functions for the chiral coefficients [18]. (2) The electromagnetic symmetry $U_{\mathrm{EM}}(1)$ is checked and found to hold in our calculation at each step. (3) Whenever $g^{\prime}$ is set to vanish, $Z$ can be expressed in terms of $W^{3}$, and similarly $W^{ \pm}$can be expressed in terms of $W^{1}$ and $W^{2}$. Thus we find that the global $S U(2)_{c}$ custodial symmetry explicitly validated in our calculation at each step. (4) When we only keep $\alpha_{0}$ in the $\beta$ functions, our results agree with those obtained by M. Tanabashi [34] and in Ref. [35]. (5) Using the alternative parameterization for Goldstone boson as

$$
U=\exp \left\{\mathrm{i} \frac{2 \xi_{3} T^{3}}{v}\right\} \exp \left\{\mathrm{i} \frac{2 \xi^{1} T^{1}+2 \xi^{2} T^{2}}{v}\right\},
$$

our computational method yields the same answer.

\section{CURRENT EXPERIMENTAL UNCERTAINTY IN $S(\Lambda)-T(\Lambda)$}

In this section we analyze the current experimental uncertainty of $S(\Lambda)-T(\Lambda)$ obtained from the constraints at the $m_{Z}$ scale listed in Table IV] First, we analyze the experimental uncertainty of $S(\Lambda)-T(\Lambda)$ induced by the uncertainty of the three-point chiral coefficients. Second, we will analyze the experimental uncertainty of $T(\Lambda)$ induced by both the uncertainty of the three-point and the four-point chiral coefficients.

Numerically, we find that $S(\Lambda), T(\Lambda)$, and $U(\Lambda)$, ( or $\alpha_{1}(\Lambda), \alpha_{0}(\Lambda)$, and $\alpha_{8}(\Lambda)$ via Eq. (40) ) are not sensitive to the running of the other chiral coefficients and that of the gauge couplings. Therefore we can use linear approximation of the RGE solutions:

$$
\begin{aligned}
& S(\Lambda)=S\left(m_{Z}\right)-\frac{2}{\pi} \beta_{\alpha_{1}} \ln \frac{\Lambda}{m_{Z}}, \\
& T(\Lambda)=T\left(m_{Z}\right)+\frac{1}{4 \pi^{2} \alpha_{\mathrm{EM}}} \beta_{\alpha_{0}} \ln \frac{\Lambda}{m_{Z}}, \\
& U(\Lambda)=U\left(m_{Z}\right)-\frac{2}{\pi} \beta_{\alpha_{8}} \ln \frac{\Lambda}{m_{Z}} .
\end{aligned}
$$

Here we explain the basic difference between the experimental uncertainty analysis of $S-T$ given in 23] and ours. The $S-T$ uncertainty contour figures given in 23] are obtained in the minimal standard model with a Higgs boson as a regulator, along with the introduction of three extra two point operators, $\overline{\mathcal{L}}_{0}, \overline{\mathcal{L}}_{1}$, and $\overline{\mathcal{L}}_{8}$, to describe the new physics effects. In this analysis, although the central values of $S, T$, and $U$ vary with the Higgs boson mass, their error bars are insensitive to $m_{H}$ and are determined by the experimental errors. To have an analogy with the prediction of the QCD-like theories, the experimental values of $S-T$ are determined by choosing $m_{H}=1 \mathrm{TeV}(1 \mathrm{TeV}$ is assumed to be the compositeness scale).

In our uncertainty analysis, we do not use Higgs as regulator but adopt the dimensional regularization and the $\overline{\mathrm{MS}}$ scheme. The uncertainties of $S\left(m_{Z}\right), T\left(m_{Z}\right)$, $U\left(m_{Z}\right)$ and their correlations are then determined by the electroweak data listed in the Table IV] Therefore, the errors at the scale $\mu=m_{Z}$ are essentially the same as those of Ref. [23]. In our analysis, however, the error bars of $S(\Lambda)-T(\Lambda)$ become larger and larger when we extrapolate the low energy constraints to high energy scale, since more and more uncertainty from the other operators creeps in the computation.

\section{A. The experimental uncertainty of $S(\Lambda)-T(\Lambda)$ from the anomalous TGC measurement}

The parameters, $S(\Lambda), T(\Lambda)$ and $U(\Lambda)$ are the values of parameters $S, T$ and $U$ at the matching scale $\Lambda$, where the EWCL matches with the underlying fundamental theories such as the QCD-like models, extra dimension models, Higgsless models, etc. In the perturbation method, $S(\Lambda), T(\Lambda), U(\Lambda)$ and $S\left(m_{Z}\right), T\left(m_{Z}\right)$, $U\left(m_{Z}\right)$ are connected by the improved renormalization group equations Eq.(61), Eq.(601), and Eq.(62), respectively. 


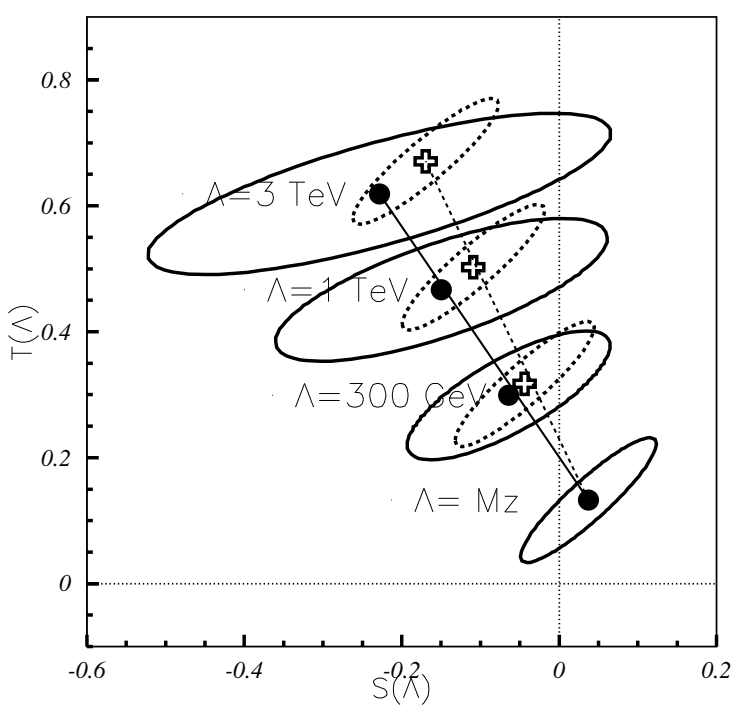

FIG. 4: The $S(\Lambda)-T(\Lambda)$ contours for various cutoff scales $\Lambda$. The solid contours are obtained when the TGC data is taken from the case 1, Eq. (48), and $U\left(m_{Z}\right)=+0.00$. The dashed contours are obtained when all the three and four point chiral coefficients are set to be zero.

With the $S, T, U$ values at $\mu=m_{Z}$ in subsection (IIIA) and the three-point chiral coefficients at $\mu=m_{Z}$ for all the three cases as given in subsection (IIIB), we evolute the values of $S, T, U$ up to the cutoff scale $\Lambda$. The results are shown in Fig. (4) for the case 1 with the constraints in Eq. (49), Fig. (5) for the case 2 with the constraints in Eq. (51), and Fig. (6) for the case 3 with the constraints in Eq. (53). In Figs. (4) and (5) for the case 1 and case 2 , we set all the coefficients that violate the custodial symmetry to zero at the scale $m_{Z}$ except $\alpha_{0}$, and therefore we use Eq. (42) for $U\left(m_{Z}\right)=0$ as the input. Because the hypercharge gauge interactions violate the custodial symmetry, we cannot impose the condistions at all the scales. According to Eq. (62), however, $U(\Lambda)$ remains negligibly small at $\Lambda=1 \mathrm{TeV}$, $U(1 \mathrm{TeV})=-0.05 \pm 0.20$ for the case 1 and $U(1 \mathrm{TeV})=$ $-0.08 \pm 0.55$ for the case 2. In Fig. (6) for the case 3, we allow all the coefficients to vary, and use Eq. (39) as the input. Results for $\Lambda$ at $300 \mathrm{GeV}, 1 \mathrm{TeV}$, and $3 \mathrm{TeV}$ are shown in these figures.

The dashed line contours correspond to the analysis without the contributions of the three-point chiral coefficients, i.e. they include only the $1 \sigma$ error of the twopoint chiral coefficients. Therefore the error contours do not change their shapes and sizes while the central values of the contours vary with the cutoff scale $\Lambda$. The solid-line contours show the analysis which includes the contributions of the three-point chiral coefficients in addition to the two point chiral coefficients.

Although the contribution of the three-point chiral co-

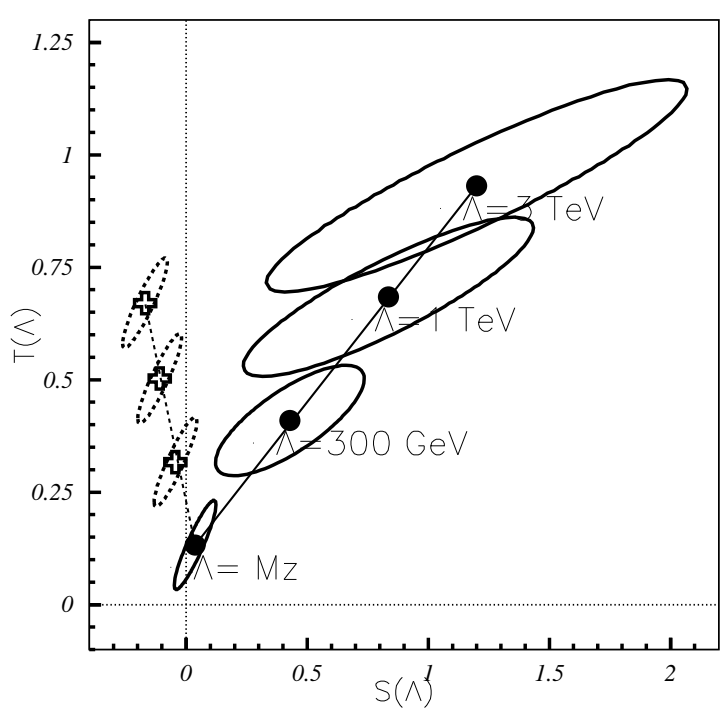

FIG. 5: The $S(\Lambda)-T(\Lambda)$ contours for various cutoff scales $\Lambda$. The solid contours are obtained when the TGC data is taken from the case 2, Eq. (50), and $U\left(m_{Z}\right)=+0.00$. The dashed contours are obtained when all the three and four point chiral coefficients are set to be zero.

efficients to $\alpha_{1}(\Lambda)-\alpha_{0}(\Lambda)$ (or $S(\Lambda)-T(\Lambda)$ ) in equations (61) and (60) are loop factor suppressed, the experimental uncertainty of these chiral coefficients is almost two orders of magnitude larger than those of the twopoint chiral coefficients, as shown in Table [IV] Therefore, if we do not make any theoretical assumptions on the magnitude of the chiral coefficients, the uncertainties of $S(\Lambda)-T(\Lambda)$ can be dominated by those of threepoint chiral coefficients at large $\Lambda$. The central values of $S(\Lambda)-T(\Lambda)$ move according to the central values of the present TGC measurements, and the size of the solid contours grows with increasing $\Lambda$.

We would like to mention some salient features with respect to Figs. 40,6 (1) In the absence of the TGC contribution (dashed line contours), $S(\Lambda)$ decreases as $\Lambda$ increases from the reference LEP1 constraint at $\Lambda=m_{Z}$. This is in agreement with the observation of Ref. 11, 22]. The dashed contours for $\Lambda=1 \mathrm{TeV}$ essentially agree with the $S-T$ contour of Ref. [23] at $m_{H}=1 \mathrm{TeV}$.

(2) When the operators that contribute to the TGC are taken into account, the $\Lambda$ dependences of the center of the $S-T$ contour are governed by the central values of the TGC measurements in Eq. (48), Eq. (50), and Eq. (52) for the case 1 (Fig. 4), the case 2 (Fig. 5), and the case 3 (Fig. 6), respectively. For instance, central value of the TGC contribution to $-\beta_{\alpha_{1}},\left(15 \alpha_{2}-5 \alpha_{3}+3 \alpha_{9}\right) g^{2} / 6$, is -0.03 for the case 1, Eq. (48), which adds up to the leading contribution of $-1 / 12$, and hence the central value of $S(\Lambda)$ gets even more negative than that of the dashed contours for $\alpha_{2}=\alpha_{3}=\alpha_{9}=0$. The central value of the 


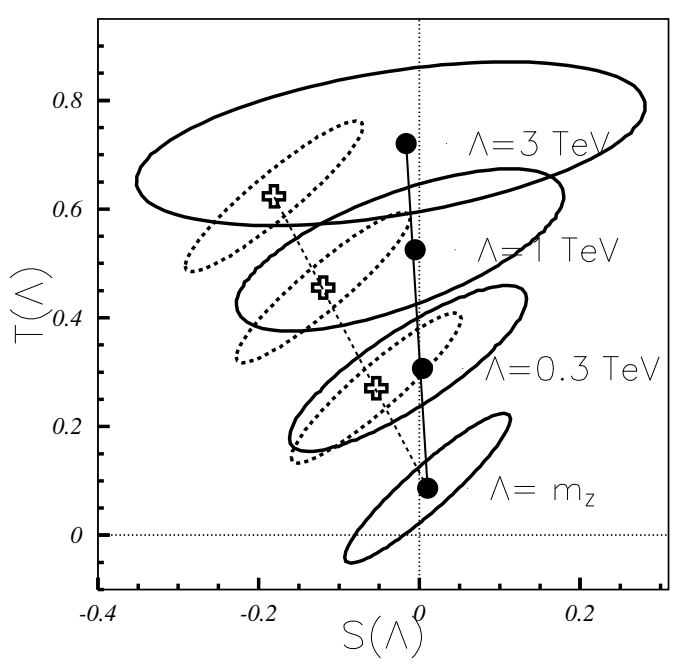

FIG. 6: The $S(\Lambda)-T(\Lambda)$ contours for various cutoff scales $\Lambda$ when the custodial symmetry is not imposed on the chiral coefficients. The solid contours are obtained when the TGC data is taken from the case 1, Eq. (52) and $S, T$, and $U$ at $\mu=m_{Z}$ are constrained by Eq. (39). The dashed contours are obtained when all the three and four point chiral coefficients are set to be zero.

same combination is 0.65 for the case 2, Eq. (50), from the L3 measurement [14], which makes the central value of $S(\Lambda)$ positive at large $\Lambda$ in Fig. 5. Large error of this single experiment constraint explains the rapid growth of the $1 \sigma$ allowed region with increasing $\Lambda$. In the case $\mathbf{3}$, where the custodial symmetry violating coefficient $\alpha_{9}$ is allowed to take an arbitrary value, the central values of Eq. (52) make the same combination about 0.08 which approximately cancels the constant term of $-1 / 12$. This results in the almost $\Lambda$-independence of the central value of $S(\Lambda)$ as observed in Fig. 6 .

In summary, those models which satisfy the uncertainty

$$
\begin{aligned}
\left(15 \alpha_{2}-5 \alpha_{3}+3 \alpha_{9}\right) g^{2} / 6 & \approx & 1.09 \alpha_{2}-0.36 \alpha_{3}+0.22 \alpha_{9} \\
& > & 1 / 12
\end{aligned}
$$

give $S(\Lambda) \geq S\left(m_{Z}\right)$, and hence QCD-like models can still be consistent with the electroweak data provided that they give rise to the TGC anomaly which satisfy the above condition. In case of the $\Lambda$-dependence of the $T$ parameter, the relevant contribution of the TGC operators in Eq. (60) gives $0.07 \alpha_{2}+0.08 \alpha_{3}-0.05 \alpha_{9}$, which tends to be smaller than the leading term of $3 g^{\prime 2} / 8$. Accordingly, in all our examples, $T(\Lambda)$ grows with increasing $\Lambda$ as can be read out from Figs. 4, 6 for the case 1, case 2 , and case 3 , respectively.

The central values of $S(\Lambda)-T(\Lambda)$ and the errors induced by TGC measurements can easily be read off from
Figs. 4,6. We find

$$
\begin{aligned}
& S(1 \mathrm{TeV})=-0.15 \pm 0.22, \\
& T(1 \mathrm{TeV})=+0.47 \pm 0.12,
\end{aligned}
$$

for the case 1, Eq. (48), with one-parameter fit data and the custodial symmetry constraint.

$$
\begin{aligned}
& S(1 \mathrm{TeV})=+0.84 \pm 0.60, \\
& T(1 \mathrm{TeV})=+0.68 \pm 0.18,
\end{aligned}
$$

for the case 2, Eq. (50), with two-parameter fit data from L3 measurement [14] and the custodial symmetry constraint, and

$$
\begin{aligned}
& S(1 \mathrm{TeV})=-0.02 \pm 0.20, \\
& T(1 \mathrm{TeV})=+0.52 \pm 0.12,
\end{aligned}
$$

for the case 3, Eq. (52), without the custodial symmetry constraint.

The central values of the above results can easily be obtained from the TGC contribution to the $\beta$ functions of $\alpha_{1}$ and $\alpha_{0}$

$$
\begin{aligned}
S(1 \mathrm{TeV})-S\left(m_{Z}\right)= & -0.13 \\
& +1.66 \alpha_{2}-0.55 \alpha_{3}+0.33 \alpha_{9}, \\
T(1 \mathrm{TeV})-T\left(m_{Z}\right)= & 0.40 \\
& +0.60 \alpha_{2}+0.70 \alpha_{3}-0.44 \alpha_{9} .
\end{aligned}
$$

These numbers and figures demonstrate an apparent fact that the uncertainty of the TGC measurement can significantly affect the value of $S(\Lambda)-T(\Lambda)$. We point out the fact that the current precision of the TGC measurement is not good enough to fix the sign of $S(\Lambda)$.

\section{B. The uncertainty of $T(\Lambda)$ from the three-point and four-point chiral coefficients}

In Figs. (44), the TGC contributions can at most modify the central value of $T(1 \mathrm{TeV})$ by $|\Delta T(1 \mathrm{TeV})| \approx$ 0.20. Thus the contribution of TGC is not large enough to cancel the large leading contribution from $3 g^{\prime 2} / 8$ in the $\beta$ function of the $\alpha_{0}$ (or equally $T$ ) parameter, which makes $T(\Lambda)$ positive.

In this section, we analyze the effect of the four-point chiral coefficients to the $T(\Lambda)$ parameter. Numerical results are given in Table $\mathrm{V}$. The columns $\delta T_{Z}$ and $\delta T^{\mathrm{TGC}}$ list the $1 \sigma$ uncertainty from the measurement of $T$ and the TGC at $\mu=m_{Z}$, respectively. The column $\delta T^{\mathrm{QGC}}$ lists the uncertainty from the QGC constrained by the theoretical unitary bounds. For the TGC constraints, we adopt the case 3, or Eq. (53) as an input.

We notice that with the increasing $\Lambda$ the TGC uncertainty $\delta T^{\mathrm{TGC}}$ increases logarithmically while the QGC uncertainty $\delta T^{\mathrm{QGC}}$ decreases rapidly. This is because of the power dependence of the unitary bounds given in Eq.(54). Consequently we find that $\delta T^{\mathrm{QGC}}>\delta T^{\mathrm{TGC}}$ for $\Lambda<950 \mathrm{GeV}$ but $\delta T^{\mathrm{QGC}}<\delta T^{\mathrm{TGC}}$ for larger $\Lambda$. 


\begin{tabular}{|l|c|c|c|c|}
\hline$\Lambda$ & $T(\Lambda) \pm 1 \sigma$ & $\delta T_{Z}$ & $\delta T^{\mathrm{TGC}}$ & $\delta T^{\mathrm{QGC}}$ \\
\hline $0.3 \mathrm{TeV}$ & $0.37 \pm 8.91$ & \pm 0.14 & \pm 0.06 & \pm 8.91 \\
$0.5 \mathrm{TeV}$ & $0.41 \pm 1.16$ & \pm 0.14 & \pm 0.08 & \pm 1.15 \\
$1 \mathrm{TeV}$ & $0.52 \pm 0.22$ & \pm 0.14 & \pm 0.12 & \pm 0.10 \\
$3 \mathrm{TeV}$ & $0.73 \pm 0.25$ & \pm 0.14 & \pm 0.17 & \pm 0.04 \\
\hline
\end{tabular}

TABLE V: Values of $T(\Lambda)$ and its uncertainty. Individual $1 \sigma$ errors from $\delta T_{Z}, \delta T^{\mathrm{TGC}}$ and $\delta T^{\mathrm{QGC}}$ are also shown.

From TableV, we can conclude that in the constrained EWCL parameter space with $1 \sigma$ error in TGC and with theoretical unitary bounds on QGC, it is unlikely to have a scenario with vanishing $T(\Lambda=1 \mathrm{TeV})$ while keeping $T\left(m_{Z}\right)=0.09$.

\section{CONCLUSIONS AND DISCUSSIONS}

In this work, we study the impacts of all the 11 dimensionless chiral coefficients $\left(\alpha_{i}, i=0, \cdots, 10\right)$ of the standard electroweak chiral Lagrangian, under the constraints from the experimental data and the perturbative unitarity bounds. We provide the improved RGEs by including the linear terms of all chiral coefficients. By using the improved RGEs, we examine the renormalization scale dependence of the oblique parameters and update the experimental uncertainty analysis on $S(\Lambda)$ and $T(\Lambda)$.

We observe that the electroweak precision measurements have constrained the oblique parameters, $S, T$, and $U$, and the anomalous TGC. According to the above experimental uncertainty analysis, we find that current precision data for the chiral coefficients of the EWCL (as given in Table IV) still allow positive $S(\Lambda)$ parameter space, as shown in Figs. (4, 6), due to the large uncertainty in the TGC chiral coefficients. Therefore, it is premature to claim that the sign of $S(\Lambda)$ conclusively rules out the QCD-like EWSB mechanism. However, the upcoming colliders, with higher sensitivity to the TGC and QGC, can further reduce the allowed parameter space and help to pinpoint the correct model of the EWSB.

Before closing, let us briefly discuss the limitations of our analysis. Most importantly, we have included neither the two-loop effects of $O\left(p^{2}\right)$ operators nor the tree-level contributions of $O\left(p^{6}\right)$ operators. In the cases when the derivative power counting rule holds, the effective couplings corresponding to the $O\left(p^{6}\right)$ operators must be suppressed by $1 /\left(16 \pi^{2}\right)^{2}$, in contrast to the $1 /\left(16 \pi^{2}\right)$ factor for the $O\left(p^{4}\right)$ operators. Meanwhile, up to $O\left(p^{6}\right)$ order, the running of $\beta_{\alpha_{i}}$ 's are not affected by the effective couplings corresponding to the $O\left(p^{6}\right)$ operators. Two loop effects of $O\left(p^{2}\right)$ operators must be suppressed by the two loop factor, and they must be tiny. Therefore, we expect that the uncertainties induced by TGC and QGC are dominant when $\Lambda=1 \mathrm{TeV}$.

We have restricted our $\beta$ functions of chiral coefficients, to retain linear terms and neglect the terms proportional to $\left(\alpha_{i} g^{2}\right)^{2}$ and $\left(\alpha_{i} g^{\prime 2}\right)^{2}$. These terms can be be classified as $p^{8}$ order effects in the derivative power counting rule as depicted in Feynman diagram FIG. (3a). Since the numerical values of $\alpha_{i}$ are small, inclusion of these terms can only render negligible correction to our $S-T$ contours.

The derivative power counting rule in the $\pi$ system has achieved great phenomenological success and its validity in the EWCL domain is yet to be tested by the experiments. From the pure theoretical viewpoint, there exist possible theories (e.g., large $N_{T C}$ theories and models with large extra dimensions) which can have large deviations in the triple and quartic gauge couplings from the prediction of the standard model. For example, in the large $N_{T C}$ theories, we can have the following power counting rules for the chiral coefficients [5]:

$$
\begin{aligned}
& \alpha_{0} \sim O\left(N_{T C}^{0}\right), \\
& \alpha_{i} \sim O\left(N_{T C}\right) .
\end{aligned}
$$

In a situation where the two-point chiral coefficients $\alpha_{0}$, $\alpha_{1}$, and $\alpha_{8}$ are already tightly constrained, one may look for symmetry breaking models which can have large triple and quartic gauge couplings.

\section{Acknowledgments}

We would like to thank Ulrich Parzefall for communication on the TGC measurements at LEP2, Masaharu Tanabashi and Masayasu Harada for stimulating discussions, and Benjamin Grinstein for communication on theoretical bounds to chiral coefficients. This work is supported in part by Grant-in-Aid for Scientific Research (\# 18340060) from Ministry of Education, Culture, Science and Technology of Japan, and the JSPS core university programs. QSY thanks the JSPS fellowship program (\# P03194) and NCTS (Hsinchu, Taiwan) for financial support. The work of $\mathrm{KY}$ is supported in part by JSPS Postdoctoral Fellowships for Research Abroad and the National Science Foundation under Grant No. NSF PHY05-51164.

\section{APPENDIX A: THE CALCULATION OF THE $\beta$ FUNCTIONS OF TWO-POINT CHIRAL COEFFICIENTS}

We describe the method of calculating the $\beta$ functions of chiral coefficients given in Eqs. (57 170 ) through the following steps [33].

1. In the background field method [36], we decompose 
fields in the Lagrangian of Eq. (3) as follows:

$$
\begin{aligned}
W & \rightarrow \bar{W}+\widehat{W} \\
B & \rightarrow \bar{B}+\widehat{B} \\
U & \rightarrow \bar{U} \widehat{U}
\end{aligned}
$$

where $\bar{W}, \bar{B}$, and $\bar{U}$ are the background fields of vector boson and background Goldstone bosons in the nonlinear realization, and $\widehat{W}, \widehat{B}$, and $\widehat{U}$ are the quantum fluctuations.

2. We parameterize $\widehat{U}$ as

$$
\widehat{U}=\exp \left\{\frac{2 \mathbf{i} \xi^{a} T^{a}}{v}\right\}
$$

and expand the Lagrangian given in Eq. (3) up to the quadratic terms

$$
\begin{aligned}
\mathcal{L}_{E W}= & \mathcal{L}_{E W}(\bar{W}, \bar{B}, \bar{U}) \\
& +\frac{\delta \mathcal{L}_{E W}}{\delta \bar{W}^{a}} \delta \widehat{W}^{a}+\frac{\delta \mathcal{L}_{E W}}{\delta \bar{B}} \delta \widehat{B} \\
& +\frac{\delta \mathcal{L}_{E W}}{\delta \xi^{a}} \xi^{a} \\
& + \text { bilinear terms of } \widehat{W}^{a}, \widehat{B}, \text { and } \xi^{a} \\
& +\cdots .
\end{aligned}
$$

The structure of the divergences upto dimension 4 operators induced by the quantum fluctuations are organized as

$$
\begin{aligned}
\delta \mathcal{L}_{E W}(\bar{W}, \bar{B}, \bar{U})= & 2 \frac{\delta g}{g} \frac{1}{g^{2}} \bar{H}_{1}+2 \frac{\delta g^{\prime}}{g^{\prime}} \frac{1}{g^{\prime 2}} \bar{H}_{2} \\
& +\frac{\delta v^{2}}{v^{2}} v^{2} \overline{\mathcal{L}}_{W / Z}+\left(\delta \alpha_{0}+\alpha_{0} \frac{\delta v^{2}}{v^{2}}\right) v^{2} \overline{\mathcal{L}}_{0} \\
& +\sum_{i=1}^{10} \delta \alpha_{i} \overline{\mathcal{L}}^{i},
\end{aligned}
$$

due to the local gauge symmetries of the classical fields, divergences of higher order operators are not relevant to our purpose and are simply thrown away.

3. We cast the quadratic terms in quantum fields ( the vector bosons $\widehat{V}^{a}$, the Goldstone bosons $\xi^{i}$, and the ghosts $v^{\bar{a}}$ and $u^{b}$ ) in a compact form with the appropriate gauge fixing terms as described in Appendix $B$ at one loop level.

$$
\mathcal{L}_{\text {quad }}=-\frac{1}{2}\left(\widehat{V}_{\mu}^{\dagger}, \xi^{\dagger}\right)\left(\begin{array}{cc}
\square_{V V}^{\mu \nu} & \overleftarrow{X}^{\mu} \\
\vec{X} & \square_{\xi \xi}^{\prime}
\end{array}\right)\left(\begin{array}{c}
\widehat{V} \\
\xi
\end{array}\right)-v^{a} \square_{v u}^{a b} u^{b} .
$$

Here we have made the partial integrals and organized the quadratic terms in the mass eigenstate basis. Calculation in the weak interaction eigenstate basis yields the same results, which serves as one extra checking point for our computation method.

4. We express the one-loop divergences induced by the quantum fluctuations by using the Gaussian integral over the $d$-dimensional space-time

$$
\begin{aligned}
\int_{x} \mathcal{L}_{1-\text { loop }}= & -\frac{1}{2}\left\{\operatorname{Tr} \ln \square_{V}+\operatorname{Tr} \ln \square_{\xi}^{\prime}-2 T r \ln \square_{v u}\right. \\
& \left.+\operatorname{Tr} \ln \left(1-\vec{X}^{\mu} \square_{V ; \mu \nu}^{-1} \overleftarrow{X}^{\nu} \square_{\xi}^{\prime-1}\right)\right\} \cdot(\mathrm{A} .8)
\end{aligned}
$$

5. Finally by using the heat kernel technique [37], we extract the divergent terms from Eq. A.8 which are linear in $\alpha_{i}$. The divergences emanating from the first three terms in Eq. (A.8) are listed in table VI, VII, and VIII respectively. Tables IX and X lists divergences contributed from the fourth term of Eq. A.8. The operator $v^{2} \mathcal{L}_{0}$ gives two terms, as shown in Eq. (A.6). The divergences listed in these tables are those of the $\delta \alpha_{0} v^{2} \mathcal{L}_{0}$, while those corresponding to $\alpha_{0} \delta v^{2} \mathcal{L}_{0}$ are neglected.

Combination of all the divergences determines the counter terms given in Eq. (A.6) and the RGE given in Eqs. (57 59). We have used the following equation of motion to remove the ambiguity of the parameterization of the Goldstone fields

$$
\begin{aligned}
\partial \cdot \bar{Z} & =\frac{O_{4}^{Z}\left(\alpha_{i}\right)}{v^{2}} \\
\mathbf{d} \cdot \bar{W}^{ \pm} & = \pm \mathbf{i} \frac{\rho g_{Z}^{2}-g^{2}}{g_{Z}} \bar{Z} \cdot \bar{W}^{ \pm}+\frac{O_{4}^{W}\left(\alpha_{i}\right)}{v^{2}},(
\end{aligned}
$$

where $\rho=1-2 \alpha_{0}$ and $d \cdot \bar{W}^{ \pm}=\partial \cdot \bar{W}^{ \pm} \mp \mathbf{i} e \bar{A} \cdot \bar{W}^{ \pm}$. The terms with $O_{4}^{Z, W}\left(\alpha_{i}\right)$ contribute to $O\left(p^{6}\right)$ and higher order operators and do not contribute to the $\beta$ functions of chiral coefficients given in Eqs. (57,70).

\section{APPENDIX B: GAUGE FIXING TERMS AND GHOST TERMS}

In order to cast the quantum fluctuations of vector boson fields into the minimal form of Eq. (A.7), we make a special choice of the gauge fixing parameters. In the background field method, the covariant gauge fixing terms are given as 


\begin{tabular}{|c|c|c|c|c|c|c|c|c|c|c|c|c|}
\hline & & $\alpha_{0}$ & $\alpha_{1}$ & $\alpha_{2}$ & $\alpha_{3}$ & $\alpha_{4}$ & $\alpha_{5}$ & $\alpha_{6}$ & $\alpha_{7}$ & $\alpha_{8}$ & $\alpha_{9}$ & $\alpha_{10}$ \\
\hline$\frac{1}{g^{2}} \bar{H}_{1}$ & $\frac{20}{3}$ & & & & $\frac{40 g^{2}}{3}$ & & & & & $4 g^{2}$ & $\frac{8 g^{2}}{3}$ & \\
\hline$\frac{1}{g^{\prime 2}} H_{2}$ & & & & & & & & & & & & \\
\hline$\overline{\mathcal{L}}_{1}$ & & & $-4 g^{2}$ & $4 g^{2}$ & $-\frac{8 g^{2}}{3}$ & & & & & & & \\
\hline $\mathcal{L}_{2}$ & & & & & & & & & & & & \\
\hline$\overline{\mathcal{L}}_{3}$ & & & & & $-\frac{20 g^{2}}{3}$ & $-2 g^{2}$ & $4 g^{2}$ & $-2 g^{2}$ & $4 g^{2}$ & & & \\
\hline $\mathcal{L}_{4}$ & & & & & & & & & & & & \\
\hline $\mathcal{L}_{5}$ & & & & & & & & & & & & \\
\hline $\mathcal{L}_{6}$ & & & & & & & & & & & & \\
\hline $\mathcal{L}_{7}$ & & & & & & & & & & & & \\
\hline $\mathcal{L}_{8}$ & & & & & & & & & & $-12 g^{2}$ & $\frac{16}{3} g^{2}$ & \\
\hline$\overline{\mathcal{L}}_{9}$ & & & & & & & & $2 g^{2}$ & $-4 g^{2}$ & & $-\frac{20 g^{2}}{3}$ & \\
\hline $\mathcal{L}_{10}$ & & & & & & & & & & & & \\
\hline$v^{2} \overline{\mathcal{L}}_{W / Z}$ & & & $2 g^{2} g^{\prime 2}$ & $-2 g^{2} g^{\prime 2}$ & $-2 g^{2} g^{\prime 2}$ & $7 g^{4}+g_{Z}^{4}$ & $10 g^{4}+4 g_{Z}^{4}$ & $g_{Z}^{4}$ & $4 g_{Z}^{4}$ & & & \\
\hline$v^{2} \overline{\mathcal{L}}_{0}$ & & & $g^{2} g^{\prime 2}$ & $-g^{2} g^{\prime 2}$ & $-g^{2} g^{\prime 2}$ & $-\frac{5}{2} g^{\prime 2}\left(g^{2}+g_{Z}^{2}\right)$ & $-g^{\prime 2}\left(g^{2}+g_{Z}^{2}\right)$ & $-g^{4}-\frac{11}{2} g_{Z}^{4}$ & $-4\left(g^{4}+g_{Z}^{4}\right)$ & & & $-6 g_{Z}^{4}$ \\
\hline
\end{tabular}

TABLE VI: Divergences contributed from $-\operatorname{Tr} \ln \square_{V} / 2$ up to linear terms of $\alpha_{i}$. The unit is $1 / 8 \pi^{2} \epsilon$.

\begin{tabular}{|c|c|c|c|c|c|c|c|c|c|c|c|c|}
\hline & & $\alpha_{0}$ & $\alpha_{1}$ & $\alpha_{2}$ & $\alpha_{3}$ & $\alpha_{4}$ & $\alpha_{5}$ & $\alpha_{6}$ & $\alpha_{7}$ & $\alpha_{8}$ & $\alpha_{9}$ & $\alpha_{10}$ \\
\hline$\frac{1}{g^{2}} \bar{H}_{1}$ & $-\frac{1}{12}$ & $\frac{1}{6}$ & & & $-\frac{g^{2}+g_{Z}^{2}}{2}$ & & & & & $-g^{2}$ & & \\
\hline$\frac{1}{g^{\prime 2}} H_{2}$ & $-\frac{1}{12}$ & $\frac{1}{3}$ & & $-g^{2}$ & & & & & & & & \\
\hline$\overline{\mathcal{L}}_{1}$ & $-\frac{1}{12}$ & & $g^{2}$ & $-\frac{g^{2}}{2}$ & $-\frac{g^{2}}{2}$ & & & & & & $-\frac{g^{2}}{2}$ & \\
\hline$\overline{\mathcal{L}}_{2}$ & $-\frac{1}{24}$ & $-\frac{1}{6}$ & & $\frac{g^{2}}{2}$ & & $\frac{g^{2}}{4}$ & $-\frac{g^{2}}{2}$ & & & & & \\
\hline$\overline{\overline{\mathcal{L}}_{3}}$ & $-\frac{1}{24}$ & $\frac{1}{6}$ & & & $\frac{g^{2}}{2}$ & $\frac{2 g^{2}+g^{\prime 2}}{8}$ & $-\frac{2 g^{2}+g^{\prime 2}}{4}$ & $\frac{2 g^{2}+g^{\prime 2}}{8}$ & $-\frac{2 g^{2}+g^{\prime 2}}{4}$ & & $-\frac{g^{2}}{2}$ & \\
\hline$\overline{\mathcal{L}_{4}}$ & $\frac{1}{12}$ & 1 & & & & $\frac{4 g^{2}+g^{\prime 2}}{2}$ & $g^{2}$ & $-\frac{g^{2}-g^{\prime 2}}{2}$ & & & & \\
\hline$\overline{\mathcal{L}_{5}}$ & $\frac{1}{12}$ & $-\frac{1}{2}$ & & & & $-\frac{6 g^{2}+g^{\prime 2}}{4}$ & $-\frac{4 g^{2}+g^{\prime 2}}{2}$ & $-\frac{g^{2}+g^{\prime 2}}{4}$ & $-\frac{3 g^{2}+g^{\prime 2}}{2}$ & & & \\
\hline$\overline{\mathcal{L}}_{6}$ & & $-\frac{3}{2}$ & & & & $\frac{g^{\prime 2}}{4}$ & $\frac{g^{\prime 2}}{2}$ & $\frac{11 g^{2}+3 g^{\prime 2}}{4}$ & $g^{2}+g^{\prime 2}$ & & & $-g^{2}+g^{\prime 2}$ \\
\hline$\overline{\mathcal{L}}_{7}$ & & $\frac{3}{4}$ & & & & $-\frac{3 g^{\prime 2}}{8}$ & $-\frac{g^{\prime 2}}{4}$ & $-\frac{9 g^{2}+7 g^{\prime 2}}{8}$ & $-\frac{g^{2}-3 g^{\prime 2}}{4}$ & & & $-2 g^{2}-g^{\prime 2}$ \\
\hline$\overline{\overline{\mathcal{L}}_{8}}$ & & $-\frac{1}{2}$ & & & $\frac{g^{\prime 2}}{2}$ & & & & & $3 g^{2}$ & $-g^{2}$ & \\
\hline$\overline{\mathcal{L}_{9}}$ & & $-\frac{1}{2}$ & & & $\frac{g^{\prime 2}}{2}$ & $-\frac{g^{\prime 2}}{8}$ & $\frac{g^{\prime 2}}{4}$ & $-\frac{2 g^{2}+g^{\prime 2}}{8}$ & $\frac{2 g^{2}+g^{\prime 2}}{4}$ & & $2 g^{2}$ & \\
\hline $\mathcal{L}_{10}$ & & & & & & & & & & & & $5 g^{2}$ \\
\hline$v^{2} \overline{\mathcal{L}}_{W / Z}$ & $\frac{\left(g^{2}+g_{Z}^{2}\right)}{4}$ & $\frac{3}{2} g^{2}-g_{Z}^{2}$ & $-\frac{g^{2} g^{\prime 2}}{2}$ & & & $-\frac{7}{4} g^{4}-\frac{1}{4} g_{Z}^{4}$ & $-\frac{5}{2} g^{4}-g_{Z}^{4}$ & $-\frac{g_{Z}^{4}}{4}$ & $-g_{Z}^{4}$ & $\frac{1}{4} g^{4}$ & & \\
\hline$v^{2} \overline{\mathcal{L}}_{0}$ & $\frac{g^{\prime 2}}{8}$ & $\frac{3}{2} g^{2}-\frac{3}{4} g_{Z}^{2}$ & $-\frac{g^{2} g^{\prime 2}}{4}$ & & & $\frac{5 g^{\prime 2}\left(g^{2}+g_{Z}^{2}\right)}{8}$ & $\frac{g^{\prime 2}\left(g^{2}+g_{Z}^{2}\right)}{4}$ & $\frac{2}{8} g^{4}+\frac{11}{8} g_{Z}^{4}$ & $g^{4}+g_{Z}^{4}$ & $\frac{g^{4}}{8}$ & & $\frac{3 g_{Z}^{4}}{2}$ \\
\hline
\end{tabular}

TABLE VII: Divergences contributed from $-\operatorname{Tr} \ln \square_{\xi} / 2$ up to linear terms of $\alpha_{i}$. The unit is $1 / 8 \pi^{2} \epsilon$.

$$
\begin{gathered}
\mathcal{L}_{G F, A}=-\frac{G_{A}}{2}\left(\partial \cdot \widehat{A}+f_{A Z} \partial \cdot \widehat{Z}-i f_{A W}\left(\widehat{W}^{-} \cdot \bar{W}^{+}-\widehat{W}^{+} \cdot \bar{W}^{-}\right)\right)^{2}, \\
\mathcal{L}_{G F, Z}=-\frac{G_{Z}}{2}\left(\partial \cdot \widehat{Z}+f_{Z \xi} \xi_{Z}+i f_{Z W}\left(\widehat{W}^{-} \cdot \bar{W}^{+}-\widehat{W}^{+} \cdot \bar{W}^{-}\right)\right)^{2}, \\
\mathcal{L}_{G F, W}=-G_{W}\left(d \cdot \widehat{W}^{+}+f_{W \xi} \xi_{W}^{+}+i f_{W Z} \bar{Z} \cdot \widehat{W}^{+}-i p_{W Z} \bar{W}^{+} \cdot \widehat{Z}+i p_{W A} \bar{W}^{+} \cdot \widehat{A}\right) \\
\left(d \cdot \widehat{W}^{-}+f_{W \xi} \xi_{W}^{-}-i f_{W Z} \bar{Z} \cdot \widehat{W}^{-}+i p_{W Z} \bar{W}^{-} \cdot \widehat{Z}-i p_{W A} \bar{W}^{-} \cdot \widehat{A}\right) .
\end{gathered}
$$

These gauge fixing terms explicitly guarantee the $U_{\mathrm{EM}}(1)$ symmetry. Gauge fixing parameters can be uniquely de- termined as follows by requiring that the quadratic terms 


\begin{tabular}{|c|c|c|c|c|c|c|c|c|c|c|c|c|}
\hline & & $\alpha_{0}$ & $\alpha_{1}$ & $\alpha_{2}$ & $\alpha_{3}$ & $\alpha_{4}$ & $\alpha_{5}$ & $\alpha_{6}$ & $\alpha_{7}$ & $\alpha_{8}$ & $\alpha_{9}$ & $\alpha_{10}$ \\
\hline$\frac{1}{a^{2}} \bar{H}_{1}$ & $\frac{2}{3}$ & & & & $\frac{2 g^{2}}{3}$ & & & & & & $\frac{g^{2}}{3}$ & \\
\hline$\frac{1}{a^{\prime 2}} H_{2}$ & & & & & & & & & & & & \\
\hline$\overline{\mathcal{L}}_{1}$ & & & & & $-\frac{g^{2}}{3}$ & & & & & & & \\
\hline $\mathcal{L}_{2}$ & & & & & & & & & & & & \\
\hline$\overline{\overline{\mathcal{L}}_{3}}$ & & & & & $-\frac{g^{2}}{3}$ & & & & & & & \\
\hline$\overline{\mathcal{L}_{4}}$ & & & & & & & & & & & & \\
\hline$\overline{\mathcal{L}_{5}}$ & & & & & & & & & & & & \\
\hline $\mathcal{L}_{6}$ & & & & & & & & & & & & \\
\hline $\mathcal{L}_{7}$ & & & & & & & & & & & & \\
\hline$\overline{\mathcal{L}}_{8}$ & & & & & & & & & & & $-\frac{g^{2}}{3}$ & \\
\hline$\overline{\mathcal{L}_{9}}$ & & & & & & & & & & & $-\frac{g^{2}}{3}$ & \\
\hline $\mathcal{L}_{10}$ & & & & & & & & & & & & \\
\hline $\bar{v}^{2} \overline{\mathcal{L}}_{W / Z}$ & & & $-2 g^{2} g^{\prime 2}$ & $g^{2} g^{\prime 2}$ & $g^{2} g^{\prime 2}$ & & & & & & & \\
\hline$v^{2} \overline{\mathcal{L}}_{0}$ & & & $-g^{2} g^{\prime 2}$ & $\frac{1}{2} g^{2} g^{\prime 2}$ & $\frac{1}{2} g^{2} g^{\prime 2}$ & & & & & & & \\
\hline
\end{tabular}

TABLE VIII: Divergences contributed from $\operatorname{Tr} \ln \square_{v u}$ up to linear terms of $\alpha_{i}$. The unit is $1 / 8 \pi^{2} \epsilon$.

\begin{tabular}{|c|c|c|c|c|c|c|c|c|c|c|}
\hline & $\alpha_{1}$ & $\alpha_{2}$ & $\alpha_{3}$ & $\alpha_{4}$ & $\alpha_{5}$ & $\alpha_{6}$ & $\alpha_{7}$ & $\alpha_{8}$ & $\alpha_{9}$ & $\alpha_{10}$ \\
\hline$\frac{1}{g^{2}} \bar{H}_{1}$ & $g^{\prime 2}$ & $-\frac{5 g^{\prime 2}}{6}$ & $-\frac{11 g^{2}}{3}+g^{\prime 2}$ & & & & & $g^{2}$ & $-\frac{5 g^{2}}{6}$ & \\
\hline$\frac{1}{g^{\prime 2}} \bar{H}_{2}$ & $2 g^{2}$ & $2 g^{2}$ & $-\frac{5 g^{2}}{3}$ & & & & & & & \\
\hline$\overline{\mathcal{L}}_{1}$ & $-g^{2}$ & $-g^{2}$ & $\frac{8 g^{2}}{3}$ & & & & & $g^{2}$ & $g^{2}$ & \\
\hline$\overline{\overline{\mathcal{L}}_{2}}$ & $\frac{g^{2}}{2}$ & $-\frac{5 g^{2}}{2}-\frac{g^{\prime 2}}{12}$ & $-\frac{5 g^{2}}{12}$ & $-\frac{g^{2}+g^{\prime 2}}{2}$ & $g^{2}-g^{\prime 2}$ & $-\frac{g^{\prime 2}}{2}$ & $-g^{\prime 2}$ & & $\frac{g^{2}}{12}$ & \\
\hline$\overline{\overline{\mathcal{L}}_{3}}$ & $\frac{g^{\prime 2}}{4}$ & $-\frac{g^{\prime 2}}{6}$ & $\frac{19 g^{2}}{12}-\frac{g^{\prime 2}}{4}$ & $\frac{g^{2}-g^{\prime 2}}{2}$ & $-g^{2}$ & $\frac{g^{2}-g^{\prime 2}}{2}$ & $-g^{2}$ & $-\frac{g^{2}}{4}$ & $\frac{13 g^{2}}{6}$ & \\
\hline$\overline{\mathcal{L}}_{4}$ & & $\frac{5 g^{\prime 2}}{6}$ & $\frac{g^{2}}{2}$ & $-\frac{15 g^{2}+13 g^{\prime 2}}{2}$ & $-3 g^{2}-3 g^{\prime 2}$ & $g^{2}-4 g^{\prime 2}$ & $-2 g^{\prime 2}$ & & $\frac{g^{2}}{6}$ & \\
\hline$\overline{\overline{\mathcal{L}}_{5}}$ & & $-\frac{g^{\prime 2}}{3}$ & $\frac{g^{2}}{2}$ & $\frac{5 g^{2}+8 g^{\prime 2}}{2}$ & $g^{2}+2 g^{\prime 2}$ & $\frac{g^{2}+2 g^{\prime 2}}{2}$ & $3 g^{2}-g^{\prime 2}$ & & $\frac{g^{2}}{3}$ & \\
\hline$\overline{\mathcal{L}}_{6}$ & & $-g^{\prime 2}$ & & $6 g^{\prime 2}$ & $3 g^{\prime 2}$ & $-\frac{18 g^{2}-g^{\prime 2}}{2}$ & $-3 g^{2}-g^{\prime 2}$ & & & $2 g^{2}-6 g^{\prime 2}$ \\
\hline$\overline{\mathcal{L}_{7}}$ & & $\frac{3 g^{\prime 2}}{4}$ & & $-\frac{19 g^{\prime 2}}{4}$ & $-\frac{5 g^{\prime 2}}{2}$ & $\frac{7 g^{2}-13 g^{\prime 2}}{4}$ & $-\frac{7 g^{2}+2 g^{\prime 2}}{2}$ & & $-\frac{g^{2}}{4}$ & $4 g^{2}-3 g^{\prime 2}$ \\
\hline$\overline{\mathcal{L}_{8}}$ & $-g^{\prime 2}$ & $\frac{5 g^{\prime 2}}{6}$ & $-g^{\prime 2}$ & & & & & $-3 g^{2}$ & $-\frac{7 g^{2}}{6}$ & \\
\hline$\overline{\overline{\mathcal{L}}_{9}}$ & $\frac{g^{\prime 2}}{4}$ & $\frac{g^{\prime 2}}{12}$ & $-\frac{7 g^{\prime 2}}{4}$ & $g^{\prime 2}$ & $g^{\prime 2}$ & $-\frac{g^{2}-2 g^{\prime 2}}{2}$ & $g^{2}+g^{\prime 2}$ & $\frac{3 g^{\prime 2}}{4}$ & $-\frac{59 g^{2}}{12}$ & \\
\hline$\overline{\mathcal{L}}_{10}$ & & & & & & $\frac{17 g^{\prime 2}}{4}$ & $\frac{7 g^{\prime 2}}{2}$ & & & $-14 g^{2}+9 g^{\prime 2}$ \\
\hline
\end{tabular}

TABLE IX: Divergences (Part A) contributed from $-\operatorname{Tr} \ln \left(1-\vec{X}^{\mu} \square_{V ; \mu \nu}^{-1} \overleftarrow{X}^{\nu} \square_{\xi}^{\prime}-1\right) / 2$ up to linear terms of $\alpha_{i}$. The unit is $1 / 8 \pi^{2} \epsilon$.

to have the minimal compact form given in Eq. (A.7):

1. The gauge parameters related with kinetic terms of the propagators of vector bosons are given as:

$$
\begin{gathered}
G_{A}=C_{1}, \\
G_{Z}=C_{3}-\frac{C_{2}^{2}}{C_{1}}, \\
G_{W}=1 .
\end{gathered}
$$

2. The gauge parameter related with the kinetic mixing between the quantum fields $\widehat{A}$ and $\widehat{Z}$ is given as

$$
f_{A Z}=\frac{C_{2}}{C_{1}^{2}} .
$$

3. The gauge parameters related with the couplings between $\widehat{A}(\widehat{Z})$ and $\widehat{W}^{+}\left(\widehat{W}^{-}\right)$are given as

$$
\begin{gathered}
f_{A W}=\frac{e}{G_{A}}, \\
f_{Z W}=\frac{1}{G_{Z}} \frac{C_{7}}{2}+\frac{e}{G_{Z}} \frac{C_{2}}{C_{1}}, \\
p_{W A}=\frac{C_{5}}{2},
\end{gathered}
$$




\begin{tabular}{|c|c|c|c|c|c|c|c|}
\hline \hline & & $\alpha_{0}$ & $\alpha_{1}$ & $\alpha_{2}$ & $\alpha_{3}$ & $\alpha_{8}$ & $\alpha_{9}$ \\
\hline$v^{2} \overline{\mathcal{L}}_{W / Z}$ & $-2 g^{2}-g^{\prime 2}$ & $-2 g^{2}+4 g^{\prime 2}$ & $2 g^{2} g^{\prime 2}$ & $-2 g^{2} g^{\prime 2}+\frac{3}{2} g^{\prime 4}$ & $-2 g^{2} g^{\prime 2}+2 g^{4}$ & $-g^{4}$ & $g^{4}-\frac{3 g^{2} g^{\prime 2}}{2}$ \\
\hline$v^{2} \overline{\mathcal{L}}_{0}$ & $-\frac{g^{\prime 2}}{2}$ & $-3 g^{2}+3 g^{\prime 2}$ & $g^{2} g^{\prime 2}$ & $-g^{2} g^{\prime 2}+\frac{3}{4} g^{\prime 4}$ & $-g^{2} g^{\prime 2}$ & $-\frac{g^{4}}{2}$ & $\frac{g^{4}}{2}-\frac{3 g^{2} g^{\prime 2}}{4}$ \\
\hline \hline
\end{tabular}

TABLE X: Divergences (Part B) contributed from $-T r \ln \left(1-\vec{X}^{\mu} \square_{V ; \mu \nu}^{-1} \overleftarrow{X}^{\nu} \square_{\xi}^{\prime}-1\right) / 2$ up to linear terms of $\alpha_{i}$. The unit is $1 / 8 \pi^{2} \epsilon$.

$$
\begin{aligned}
& p_{W Z}=\frac{C_{6}}{2}, \\
& f_{W Z}=\frac{C_{7}}{2} .
\end{aligned}
$$

These gauge parameters guarantee that the covariant differential operator of vector sector has an Hermitian form.

4. The gauge parameters related with the mixing between the quantum vector bosons and the Goldstone bosons are determined as

$$
\begin{aligned}
f_{Z \xi} & =\frac{\rho}{G_{Z}} \frac{g_{Z} v}{2}, \\
f_{W \xi} & =-\frac{1}{G_{W}} \frac{g v}{2} .
\end{aligned}
$$

The parameter $f_{Z \xi}$ takes into account the diagonalization and the normalization of the $Z$ vector boson and the $\xi_{Z}$ Goldstone boson.

Here, the parameters $C_{i}$ are defined as

$$
\begin{gathered}
C_{1}=1-\frac{g^{2} g^{\prime 2}}{g_{Z}^{2}}\left(2 \alpha_{1}+\alpha_{8}\right), \\
C_{2}=\frac{g g^{\prime}}{g_{Z}^{2}}\left[\alpha_{1}\left(g^{2}-g^{\prime 2}\right)+\alpha_{8} g^{2}\right], \\
C_{3}=1+\frac{g^{2}}{g_{Z}^{2}}\left(2 \alpha_{1} g^{\prime 2}-\alpha_{8} g^{2}\right),
\end{gathered}
$$

$$
\begin{aligned}
C_{5}= & 2 \frac{g g^{\prime}}{g_{Z}}\left[1-\left(\alpha_{1}+\alpha_{8}\right.\right. \\
& \left.\left.-\alpha_{2}-\alpha_{3}-\alpha_{9}\right) g^{2}\right],
\end{aligned}
$$

$$
\begin{aligned}
C_{6}= & 2 \frac{g^{2}}{g_{Z}}\left[1-\left(\alpha_{8}-\alpha_{3}-\alpha_{9}\right) g^{2}\right. \\
& \left.+\left(\alpha_{1}-\alpha_{2}\right) g^{\prime 2}\right]
\end{aligned}
$$

$$
C_{7}=2 \frac{g^{2}}{g_{Z}}\left(1+\alpha_{3} g_{Z}^{2}\right) .
$$

From the gauge fixing terms given in Eqs. B.1,B.3), the determinant of the ghost terms are found as

$$
\operatorname{det}\left(\frac{\delta F}{\delta \alpha}\right)=\operatorname{det}\left(D^{\prime} \cdot D+\frac{\delta F}{\delta \xi} \frac{\delta \xi}{\delta \alpha}\right)
$$

where $i D_{\mu}^{\prime}$ is non-Hermitian with

$$
D_{\mu}^{\prime}=C_{g h} \partial_{\mu}+\Gamma_{\mu}^{\prime},
$$

where the matrix $C_{g h}$ is

$$
\mathbf{C}_{\mathbf{g h}}=\left(\begin{array}{cccc}
\sqrt{G_{A}} & \sqrt{G_{A}} f_{A Z} & 0 & 0 \\
0 & \sqrt{G_{Z}} & 0 & 0 \\
0 & 0 & 1 & 0 \\
0 & 0 & 0 & 1
\end{array}\right)
$$

and the matrix $\Gamma^{\prime}$ is

$$
\Gamma^{\prime}=\left(\begin{array}{cccc}
0 & 0 & i \sqrt{G_{A}} f_{A W} \bar{W}^{-} & -i \sqrt{G_{A}} f_{A W} \bar{W}^{+} \\
0 & 0 & -i \sqrt{G_{Z}} f_{Z W} \bar{W}^{-} & i \sqrt{G_{Z}} f_{Z W} \bar{W}^{+} \\
i p_{W A} \bar{W}^{+} & -i p_{W Z} \bar{W}^{+} & -i e \bar{A}+i f_{W Z} \bar{Z} & 0 \\
-i p_{W A} \bar{W}^{-} & i p_{W Z} \bar{W}^{-} & 0 & i e \bar{A}-i f_{W Z} \bar{Z}
\end{array}\right)
$$

Here we observe that Hermiticity of this determinant is broken by both the kinetic parameter $C_{g h}$ and the gauge potential terms $\Gamma^{\prime}$ by the chiral coefficients $\alpha_{i}$. The parameter $C_{g h}$ is determined by the chiral coefficients $\alpha_{1}$ 
and $\alpha_{8}$, which induce the mixing between photon and the $\mathrm{Z}$ boson. In $\Gamma^{\prime}$, Hermiticity is broken by the chiral coef- ficients $\alpha_{2}, \alpha_{3}$, and $\alpha_{9}$. On the other hand, $D=\partial+\Gamma$ is Hermitian and $\Gamma$ is given as

$$
\left(\begin{array}{cccc}
0 & 0 & i e \bar{W}^{-} & -i e \bar{W}^{+} \\
0 & 0 & -i \frac{g^{2}}{g_{Z}} \bar{W}^{-} & i \frac{g^{2}}{g_{Z}} \bar{W}^{+} \\
i e \bar{W}^{+} & -i \frac{g^{2}}{g_{Z}} \bar{W}^{+} & -i e \bar{A}+i \frac{g^{2}}{g_{Z}} \bar{Z} & 0 \\
-i e \bar{W}^{-} & i \frac{g^{2}}{g_{Z}} \bar{W}^{-} & 0 & i e \bar{A}-i \frac{g^{2}}{g_{Z}} \bar{Z}
\end{array}\right)
$$

The term $(\delta F / \delta \xi)(\delta \xi / \delta \alpha)$ is

$$
\left(\begin{array}{cccc}
0 & 0 & 0 & 0 \\
0 & -\frac{\rho}{\sqrt{G_{Z}}} \frac{g_{Z}^{2} v^{2}}{4} & 0 & 0 \\
0 & 0 & -\frac{g^{2} v^{2}}{4} & 0 \\
0 & 0 & 0 & -\frac{g^{2} v^{2}}{4}
\end{array}\right)
$$

We can make the ghost determinant to be Hermitian, by using the fact that

$$
\operatorname{det}\left(\frac{\delta F^{a}}{\delta \alpha^{\bar{b}}}\right)=\frac{\operatorname{det}\left(M^{a b} \frac{\delta F^{b}}{\delta \alpha^{a}} \bar{M}^{\bar{a} \bar{b}}\right)}{\operatorname{det}\left(M^{a b}\right) \operatorname{det}\left(\bar{M}^{\bar{a} \bar{b}}\right)} .
$$

This identity is justified if $\operatorname{det} M^{a b}$ and $\operatorname{det} \bar{M}^{\bar{a} \bar{b}}$ do not vanish or go to infinity. In the ghost term, the degree of freedom to choose the matrices $M^{a b}$ and $\bar{M}^{\bar{a} \bar{b}}$ reflects the fact that there are two types of real ghost fields we can introduce, which are labeled as $v$ type and $u$ type ghosts, respectively.

By properly choosing the matrices $M^{a b}$ and $\bar{M}^{\bar{a} \bar{b}}$, we can reorganize the ghost determinant as

$$
\begin{aligned}
\operatorname{det}\left(M^{a b} \frac{\delta F^{b}}{\delta \alpha^{\bar{a}}} \bar{M}^{\bar{a} \bar{b}}\right)= & D_{g h} \cdot D_{g h} \\
& +\sigma_{g h}^{\text {mass }}+\sigma_{g h}^{2},
\end{aligned}
$$

where $D_{g h}$ is Hermitian. The mass matrix $\sigma_{g h}^{\text {mass }}$ is the same as that of the vector bosons. There are several ways to adjust the matrices $M^{a b}$ and $\bar{M}^{\bar{a} \bar{b}}$ in order to make the Feynman rules of the ghost sector well-defined. Although we cannot make the term $\sigma_{g h}^{2}$ Hermitian, up to linear terms of $\alpha_{i}$, we find our results given in Table VIII is independent of the procedures to hermitize the ghost determinant.
[1] S. Weinberg, Phys. Rev. D 13 (1976) 974; Phys. Rev. D 19 (1979) 1277; L. Susskind, Phys. Rev. D 20 (1979) 2619;

[2] C. T. Hill and E. H. Simmons, Phys. Repts. 381 (2003) 235 [ Erratum-ibid. 390 (2004) 553].

[3] S. Dutta, K. Hagiwara, Q.S. Yan, unpublished, arXiv:hep-ph/0603038 Q.S. Yan, arXiv:hep-ph/0703189, talk presented at SCGT2006 workshop, Nagoya, Japan (November 2006).

[4] J. Gasser and H. Leutwyler, Annals Phys. 158 (1984) 142 ;

[5] J. Gasser and H. Leutwyler, Nucl. Phys. B 250 (1985) 465.

[6] M. Harada and K. Yamawaki, Phys. Rept. 381 (2003) 1 arXiv:hep-ph/0302103.

[7] T. Appelquist and C. W. Bernard, Phys. Rev. D 22, 200 (1980); Phys. Rev. D 23, 425 (1981).

[8] A. C. Longhitano, Phys. Rev. D 22, 1166 (1980).

[9] A. C. Longhitano, Nucl. Phys. B 188, 118 (1981).

[10] T. Appelquist and G. H. Wu, Phys. Rev. D 48, 3235 (1993) arXiv:hep-ph/9304240.

[11] J. A. Bagger, A. F. Falk and M. Swartz, Phys. Rev. Lett. 84, 1385 (2000) hep-ph/9908327.
[12] A. Heister et al. [ALEPH Collaboration], Eur. Phys. J. C 21, 423 (2001) arXiv:hep-ex/0104034.

[13] G. Abbiendi et al. [OPAL Collaboration], Eur. Phys. J. C 33, 463 (2004) arXiv:hep-ex/0308067.

[14] P. Achard et al. [L3 Collaboration], Phys. Lett. B 586, 151 (2004) arXiv:hep-ex/0402036.

[15] S. Schael et al. [ALEPH Collaboration], Phys. Lett. B 614, 7 (2005).

[16] V. M. Abazov et al. [D0 Collaboration], arXiv:hep-ex/0504019.

[17] H. Georgi, Annu. Rev. Nucl. Part. Sci. 43 (1993) 209.

[18] M. J. Herrero and E. Ruiz Morales, Nucl. Phys. B 418, 431 (1994) arXiv:hep-ph/9308276]; Nucl. Phys. B 437, 319 (1995) arXiv:hep-ph/9411207]; S. Dittmaier and C. Grosse-Knetter, Nucl. Phys. B 459, 497 (1996) arXiv:hep-ph/9505266.

[19] R. Barbieri et. al. , Nucl. Phys. B 703, 127 (2004).

[20] P. Sikivie, L. Susskind, M. Voloshin and V. Zakharov, Nucl. Phys. B173, 189 (1980).

[21] M. E. Peskin and T. Takeuchi, Phys. Rev. Lett. 65, 964 (1990); Phys. Rev. D46, 381 (1992).

[22] M. E. Peskin and J. D. Wells, Phys. Rev. D 64, 093003 (2001). 
[23] W. M. Yao et al. [Particle Data Group], J. Phys. G 33, 1 (2006).

[24] [ALEPH Collaboration], Phys. Rept. 427, 257 (2006) arXiv:hep-ex/0509008.

[25] K. Hagiwara, S. Matsumoto, D. Haidt and C. S. Kim, Z. Phys. C 64, 559 (1994) [Erratum-ibid. C 68, 352 (1995)] arXiv:hep-ph/9409380; K. Hagiwara, D. Haidt and S. Matsumoto, Eur. Phys. J. C 2, 95 (1998) arXiv:hep-ph/9706331. K. Hagiwara, Annu. Rev. Nucl. Part. Sci. 48 463, (1998).

[26] D. Y. Bardin, P. Christova, M. Jack, L. Kalinovskaya, A. Olchevski, S. Riemann and T. Riemann, Comput. Phys. Commun. 133, 229 (2001) arXiv:hep-ph/9908433; A. B. Arbuzov et al., Comput. Phys. Commun. 174, 728 (2006) arXiv:hep-ph/0507146.

[27] E. Brubaker et al. TTevatron Electroweak Working Group], arXiv:hep-ex/0608032

[28] K. Hagiwara, R. D. Peccei, D. Zeppenfeld and K. Hikasa, Nucl. Phys. B 282, 253 (1987).

[29] U. Baur and D. Zeppenfeld, Phys. Lett. B 201, 383 (1988).

[30] LEPEWWG/TGC/2005-01, WWW access at
http://www.cern.ch/LEPEWWG/lepww/tgc

[31] J. M. Cornwall, D. N. Levin and G. Tiktopoulos, Phys. Rev. Lett. 30, 1268 (1973) [Erratum-ibid. 31, 572 (1973)]. J. M. Cornwall, D. N. Levin and G. Tiktopoulos, Phys. Rev. D 10, 1145 (1974) [Erratum-ibid. D 11, 972 (1975)]. C. H. Llewellyn Smith, Phys. Lett. B 46, 233 (1973). S. D. Joglekar, Annals Phys. 83, 427 (1974). B. W. Lee, C. Quigg and H. B. Thacker, Phys. Rev. D 16, 1519 (1977). B. W. Lee, C. Quigg and H. B. Thacker, Phys. Rev. Lett. 38, 883 (1977).

[32] J. Distler, B. Grinstein, R. A. Porto and I. Z. Rothstein, Phys. Rev. Lett. 98, $041601 \quad$ (2007) arXiv:hep-ph/0604255.

[33] Q. S. Yan and D. S. Du, Phys. Rev. D 69, 085006 (2004); S. Dutta, K. Hagiwara, and Q. S, Yan, Nuc. Phys. B 704, 75 (2005).

[34] M. Tanabashi, private communication.

[35] R. S. Chivukula, S. Matsuzaki, E. H. Simmons and M. Tanabashi, arXiv:hep-ph/0702218

[36] L. F. Abbott, Acta Phys. Polon. B 13 (1982) 33.

[37] D. V. Vassilevich, Phys. Rept. 388, 279 (2003). 